\title{
Multi-scale wave propagation modelling for two-dimensional periodic textile composites
}

\author{
V. Thierry, L. Brown, D.Chronopoulos ${ }^{1}$ \\ Institute for Aerospace Technology \& The Composites Research Group, The University of Nottingham, NG7 2RD, UK
}

\begin{abstract}
In this article, a method allowing vibro-acoustic and ultrasonic wave propagation analysis of highly anisotropic textile composites at a mesoscopic level is presented for the first time. The method combines the advantages of mode-based Component Mode Synthesis (CMS) that allows reduction of the size of the Dynamic Stiffness Matrix (DSM) of a textile unit cell, and of a Wave Finite Element Method (WFEM), which associates the Periodic Structure Theory (PST) with standard Finite Element Method (FEM). The scheme presented allows the study of the wave propagation properties of a periodic structure by modelling only a unit cell. A multi-scale approach is used to enable the comparison of standard vibrational analysis of textile composite structures, using homogenized properties, with a more complex analysis, where the mesoscale properties of the structure are preserved. It is shown for two different types of weaves that using a standard homogenised model results in significant errors in the dispersion curves. Also band-gap behaviour within specific frequency ranges are successfully predicted using the mesoscale models, whereas it was not observed in the macroscale ones.

Keywords: Textile composite structures, Wave propagation, Periodic Structure Theory, Component Mode Synthesis, Unit cell modelling, Multiscale homogenization
\end{abstract}

\section{Introduction}

Textile composites are increasingly used in modern industry due to their excellent mechanical properties (high specific stiffness and strength, superb fatigue strength, excellent corrosion resistance and dimensional stability [1]) and especially for aerospace applications thanks to a lower weight than metallic alloys. These composite structures can be seen as an infinite assembly of identical elements called unit cells joined in an identical manner. The wide application of these periodic structures have attracted a lot of research for more than a decade $[2,3,4,5,6]$.

All man-made structures have finite life spans and begin to degrade as soon as they are put in service. Textile composites in particular are prone to many modes of failures such as fibre breakages, cracks and delamination. In the process of Structural Health Monitoring (SHM) - which consists of performing Non Destructive Evaluation (NDE) by the means of sensor integration, possibly smart materials, data transmission, computational modelling, and processing ability inside the structures [7] - the earliest possible detection of damage is important. Indeed, in the aerospace industry, damage can lead to catastrophic failures. At present, approximately $27 \%$ of an average aircraft's life cycle 
cost is spent on maintenance [8]. The use of 'on the ground' structural inspection techniques leads to a massive reduction of the aircraft's availability and thus significant financial losses for the operator. That is why intensive research is conducted for 'on-line' evaluation of damage in industrial structural components. A technique finding increasing popularity for 'on-line' inspection is ultrasound guided waves spectroscopy [9]. As guided wave propagation in thin plates is dispersive, the velocity of wave propagation (and thus the time-of-flight) depends on the frequency. Therefore it is of the most importance to know the dispersion relations for use in NDE and SHM.

For these reasons, numerical simulations are increasingly needed for the design and optimization of inspection processes for textile composite aerospace structures. Different modelling scales can be combined in order to study the systems response to different frequency bands interrogation. Indeed, in the low frequency range, the vibrational behaviour of periodic structures is governed by the macroscopic material parameters (the unit cell can be modelled by a low number of elements symbolising the homogeneous material parameters). For this frequency range there will be no local resonance; it will rather involve vibration of the entire structural component and therefore this would not be suitable for the detection of small damages [7]. In the mid-frequency range, the mechanical energy propagates in the entire structure and produces wave localization in the periodic cell, with the interrogation of the structure in this range of frequency being more suitable for the detection of small cracks. In order to study this frequency range, the unit cell needs to be modelled at a mesoscopic scale. Finally, in the high frequency range where local resonances and Bragg scattering phenomena are intense, the mechanical energy mainly remains in the periodic cell, and thus is not interesting for this kind of study [6].

The Wave Finite Element Method (WFEM) is an efficient tool to study wave propagation in periodic structures. The WFEM has been applied in numerous previous works such as beam-like structures in its one-dimensional formulation $[10,11]$ and then the method was extended to two-dimensional by [12] and applied to various type of structures such as cylinders [12, 13], plates [14, 15], thin-walled structures [16] and curved layered shells [17]. However, to apply the WFEM to textile composites, a fine mesh is needed to describe the geometry precisely, which implies large number of degrees of freedom and therefore large CPU times. Various model order reduction (MOR) strategies have been investigated in order to counteract this issue. One of them is the component mode synthesis (CMS) approach, which has been widely used in the literature $[6,18,19,20,21]$. Other techniques exist such as the model reduction strategy introduced in [22], based on the selection of the contributing waves or the strategy developed in [23] which relies on frequency interpolation of the transfer matrix eigenvectors.

Wave propagation in periodic structures has been studied for a long time with some iconic works throughout the last two centuries [3, 24, 25, 26]. A well-known characteristic of periodic structures is that they can exhibit wave filtering properties. These are called 'stop-bands'. They are activated by local resonances or Bragg scatterings $[21,27,28]$. When it exists, the propagation of certain waves is forbidden within some frequency ranges. This unique property is useful in many applications such as acoustic wave filtering or vibration isolation [28, 29, 30, 31, 32]. But often is a disadvantage for guided wave inspection as it restricts the use of some modes for this application [31, 33]. Predicting stop-band behaviour is thus of great interest and it has been widely investigated for metamaterials such as 
phononic crystal $[30,34,35]$ or composite materials [27, 29, 32, 36]. However little research has been conducted in textile composites with strongly anisotropic structure.

The principal novelty of this work is the investigation of ultrasonic wave propagation analysis in highly anisotropic periodic structures such as textile composites. The mesoscopic properties of the material are taken into account for the first time for this type of analysis. It is shown that standard homogenised composite structures calculations are wrong and that mesoscale modelling is needed. The most emphatic difference is that mesoscale models can successfully predict stop-band behaviour which is indispensable for guided wave inspection purposes. It should however be indicated that the presented formulation cannot capture size effects in nano-structural analyses [37, 38, 39].

This paper is organised as follows. In section 2, an overview of the method used to model the textile composite structure is shown. Then the wave and finite element method will be described, coupled with a model reduction method, both used for the ultrasound wave propagation analysis of composite structures in this paper. Finally, examples of vibrational analyses of different composite textiles will be presented.

\section{Multiscale modelling of textile composites}

The global methodology used in this paper is summarized in Fig.1.

Mechanical modelling of textile composites can be achieved at different scales [40]. A macroscale modelling would imply homogenised mechanical properties along the unit cell, while a mesoscale modelling suggests a more detailed geometric representation of fabrics such as the yarns and the matrix (in this case, the assembly of fibres are generally homogenised). Finally, a microscopic modelling indicates not only the representation of the yarns and the matrix, but also the assembly of fibres that compose each yarn. In this paper, the investigations are carried out at two scales: macro- and mesoscales.

\subsection{Mesoscale modelling}

Mesoscale modelling of textile composites begins with the definition of textile unit cell geometry using a specialist pre-processor such as Texgen [41, 42, 43, 44, 45]. This open source software is developed by the Composites Research Group at the University of Nottingham and is used for modelling the geometry of textile structures such as 2D or 3D woven textiles (see Fig.2). The mechanical properties are added to the yarns and the matrix during the model definition process. Once the model has been generated, it can be exported to a FE software, and finally the mass and stiffness matrices $\mathbf{M}$ and $\mathbf{K}$ of the unit cell can be extracted, as shown in Fig.1.

\subsection{From meso- to macroscale modelling}

A macroscale model is defined by its homogenised mechanical properties. Traditionally, material characterisation refers to material testing as a way to obtain the material properties. The establishment of a unit cell is an alternative for acquiring those properties, even though it does not replace a physical testing completely. This method can be seen as virtual testing of the material. The analyses are conducted on the mesoscale model in order to evaluate the effective 


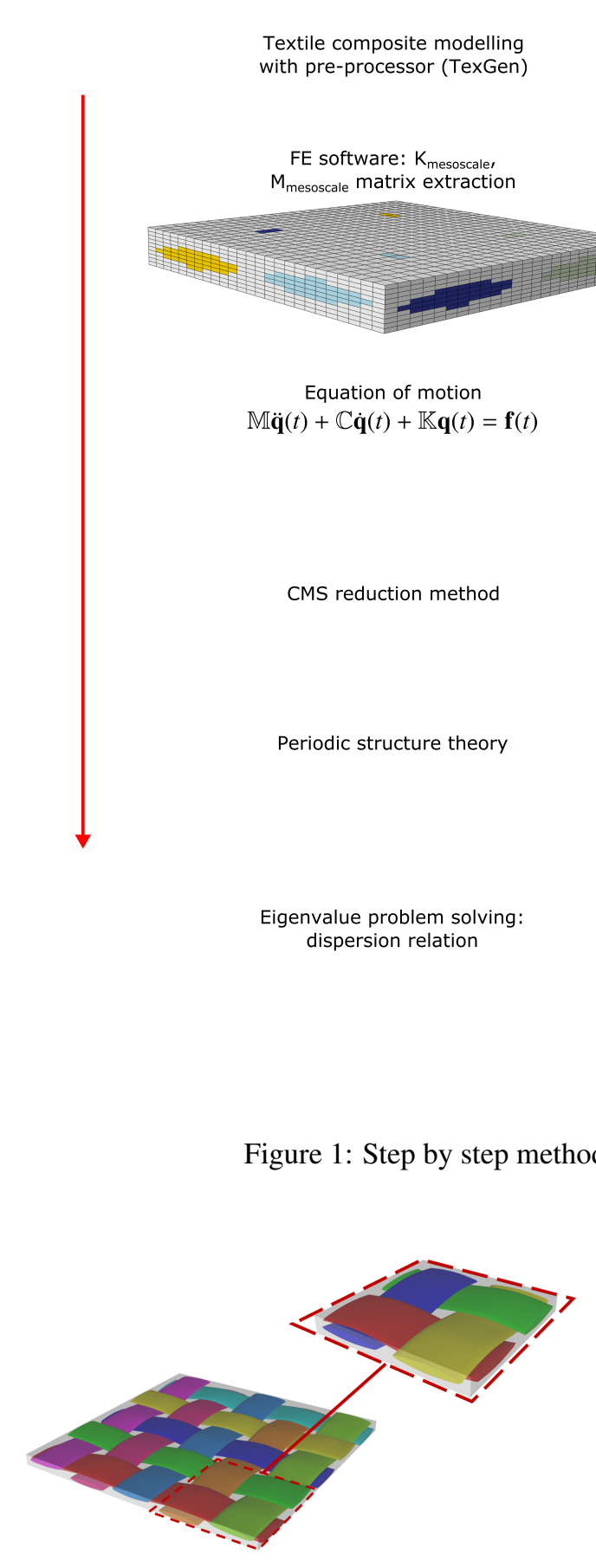

(a) Unit cell of a periodic 2D weave

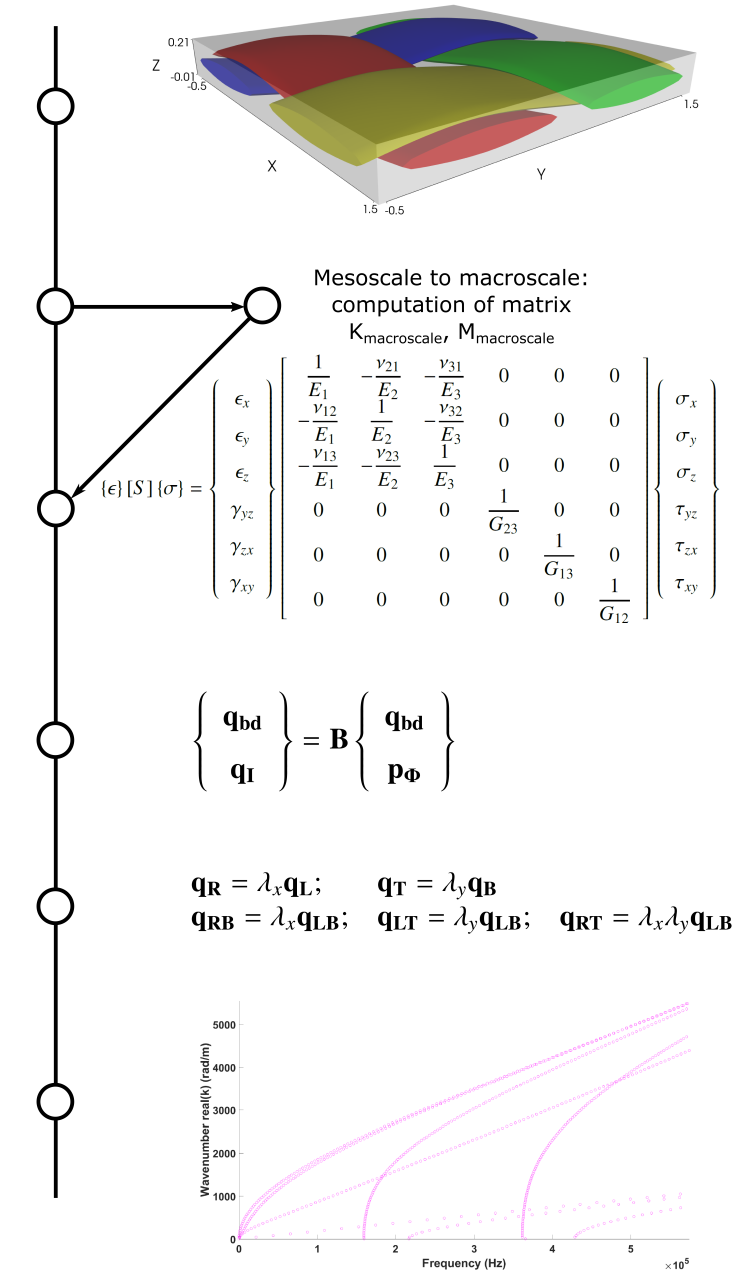

$\begin{array}{ll}\mathbf{q}_{\mathbf{R}}=\lambda_{x} \mathbf{q}_{\mathbf{L}} ; & \mathbf{q}_{\mathbf{T}}=\lambda_{y} \mathbf{q}_{\mathbf{B}} \\ \mathbf{q}_{\mathbf{R B}}=\lambda_{x} \mathbf{q}_{\mathbf{L B}} ; & \mathbf{q}_{\mathbf{L T}}=\lambda_{y} \mathbf{q}_{\mathbf{L}}\end{array}$

Figure 1: Step by step methodology of the presented mesoscale formulation

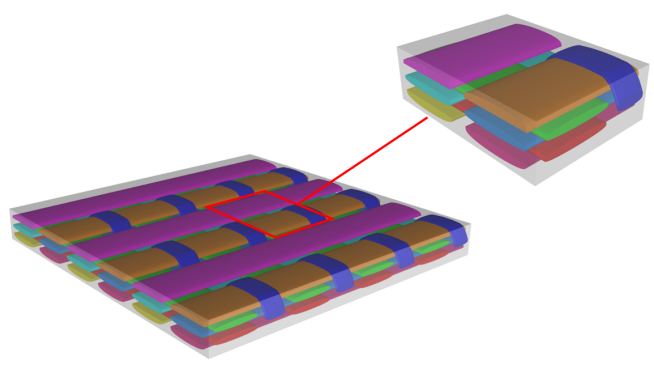

(b) Unit cell of a periodic 3D weave

Figure 2: Two examples of periodic textile composites and the corresponding unit cells 
material properties of the macroscale model. Some basic assumptions are that the macroscale model is effectively homogeneous (in this case the periodicity of the structure at the mesoscale can justify this assumption) and the stress and strain states imposed to it are uniform [46]. It is important to note that according to the periodic pattern, the boundary conditions are different for an identic unit cell. In this paper, the periodic boundary condition equations are generated based on the method developed in $[47,48,49,46]$. As can be observed in Fig.2, the periodic pattern is formed by translational symmetries of the considered unit cell along $x$ - and $y$-axis.

\subsubsection{Displacement boundary conditions for unit cells}

The boundary conditions must be given for each pair of faces of the unit cell. The considered textile can be seen as a simple cubic packing of cells as described in [47], only without periodicity along the $z$-axis in our case. $2 b_{x}, 2 b_{y}$ and $2 b_{z}$ (see Fig.3) give the dimensions of the unit cell in the $x, y$ and $z$ directions respectively. This implies that any point $P^{\prime}\left(x^{\prime}, y^{\prime}, z^{\prime}\right)$ in the textile outside the considered unit cell has an image $P(x, y, z)$ in the unit cell:

$$
\left(x^{\prime}, y^{\prime}, z^{\prime}\right)=\left(x+2 \mathrm{i} b_{x}, y+2 \mathrm{j} b_{y}, z\right)
$$

where $i$ and $j$ are the number of unit cells separating $P^{\prime}$ from $P$ in the $x$ and $y$ directions, respectively.

The relative displacement between $P$ and $P^{\prime}$ in the mesoscale unit cell (displacement noted as $(u, v, w)$ ) must be the same as the relative displacement between those same points in the macroscale unit cell (displacement noted as $(U, V$, $W)$ ) [46], i.e.,

$$
\left.\left\{\begin{array}{c}
u \\
v \\
w
\end{array}\right\}\right|_{P^{\prime}}-\left.\left\{\begin{array}{c}
u \\
v \\
w
\end{array}\right\}\right|_{P}=\left.\left\{\begin{array}{c}
U \\
V \\
W
\end{array}\right)\right|_{P^{\prime}}-\left.\left\{\begin{array}{c}
U \\
V \\
W
\end{array}\right)\right|_{P}=\left\{\begin{array}{c}
\Delta U \\
\Delta V \\
\Delta W
\end{array}\right\}
$$

The relative displacement field in the macroscale model can be written as [46]:

$$
\left\{\begin{array}{c}
\Delta U \\
\Delta V \\
\Delta W
\end{array}\right\}=\left[\begin{array}{ccc}
\frac{\partial U}{\partial x} & \frac{\partial U}{\partial y} & \frac{\partial U}{\partial z} \\
\frac{\partial V}{\partial x} & \frac{\partial V}{\partial y} & \frac{\partial V}{\partial z} \\
\frac{\partial W}{\partial x} & \frac{\partial W}{\partial y} & \frac{\partial W}{\partial z}
\end{array}\right]\left\{\begin{array}{c}
\Delta x \\
\Delta y \\
\Delta z
\end{array}\right\}
$$

In order to eliminate rigid body motions, the displacement at an arbitrary point (we choose $\mathrm{O}$ whose coordinates are $(0,0,0))$ are suppressed and the rotations of the $x$-axis about the $y$-and $z$-axis, respectively, and that of the $y$-axis about the $x$-axis are constrained at that same point $\mathrm{O}$ as follows $[47,48,49,46]$ :

$$
\frac{\partial V}{\partial x}=\frac{\partial W}{\partial x}=\frac{\partial W}{\partial y}=0
$$


It is important to clarify that this is not a unique expression for constraining the rigid body rotation, there are multiple ways as presented in [46]. This is however the most convenient and thus the one being used in this paper [46]. Finally, the relative displacement field becomes

$$
\left.\left\{\begin{array}{c}
u \\
v \\
w
\end{array}\right\}\right|_{P^{\prime}}-\left.\left\{\begin{array}{c}
u \\
v \\
w
\end{array}\right)\right|_{P}=\left[\begin{array}{ccc}
\frac{\partial U}{\partial x} & \frac{\partial U}{\partial y} & \frac{\partial U}{\partial z} \\
0 & \frac{\partial V}{\partial y} & \frac{\partial V}{\partial z} \\
0 & 0 & \frac{\partial W}{\partial z}
\end{array}\right]\left\{\begin{array}{c}
\Delta x \\
\Delta y \\
\Delta z
\end{array}\right\}=\left[\begin{array}{ccc}
\epsilon_{x}^{0} & \gamma_{x y}^{0} & \gamma_{x z}^{0} \\
0 & \epsilon_{y}^{0} & \gamma_{y z}^{0} \\
0 & 0 & \epsilon_{z}^{0}
\end{array}\right]\left\{\begin{array}{c}
\Delta x \\
\Delta y \\
\Delta z
\end{array}\right\}
$$

$\epsilon_{x}^{0}, \epsilon_{y}^{0}, \epsilon_{z}^{0}, \gamma_{x y}^{0}, \gamma_{y z}^{0}, \gamma_{z x}^{0}$ being the macroscopic strains.

As a result, the faces of the unit cells in this packing configuration are defined by the following translational symmetry transformations: for the parts of the boundary normal to the $x$-axis (see faces A and B in Fig.3), the translation is given as

$$
\left\{\begin{array}{c}
\Delta x \\
\Delta y \\
\Delta z
\end{array}\right\}=\left\{\begin{array}{c}
2 b_{x} \\
0 \\
0
\end{array}\right\}
$$

Using Eq.5, the relative displacement boundary conditions is obtained

$$
\begin{aligned}
& \left(\left.u\right|_{x=b_{x}}-\left.u\right|_{x=-b_{x}}\right)=2 b_{x} \epsilon_{x}^{0} \\
& \left(\left.v\right|_{x=b_{x}}-\left.v\right|_{x=-b_{x}}\right)=0 \\
& \left(\left.w\right|_{x=b_{x}}-\left.w\right|_{x=-b_{x}}\right)=0
\end{aligned}
$$

Similarly for the pair of faces normal to $y$-axis (faces C and D),

$$
\left\{\begin{array}{c}
\Delta x \\
\Delta y \\
\Delta z
\end{array}\right\}=\left\{\begin{array}{c}
0 \\
2 b_{y} \\
0
\end{array}\right\}
$$

and hence

$$
\begin{aligned}
& \left(\left.u\right|_{y=b_{y}}-\left.u\right|_{y=-b_{y}}\right)=2 b_{y} \gamma_{x y}^{0} \\
& \left(\left.v\right|_{y=b_{y}}-\left.v\right|_{y=-b_{y}}\right)=2 b_{y} \epsilon_{y}^{0} \\
& \left(\left.w\right|_{y=b_{y}}-\left.w\right|_{y=-b_{y}}\right)=0
\end{aligned}
$$


Finally, the conditions can be written similarly for the pair of faces normal to z-axis,

$$
\begin{aligned}
& \left(\left.u\right|_{z=b_{z}}-\left.u\right|_{z=-b_{z}}\right)=2 b_{z} \gamma_{x z}^{0} \\
& \left(\left.v\right|_{z=b_{z}}-\left.v\right|_{z=-b_{z}}\right)=2 b_{z} \gamma_{y z}^{0} \\
& \left(\left.w\right|_{z=b_{z}}-\left.w\right|_{z=-b_{z}}\right)=2 b_{z} \epsilon_{z}^{0}
\end{aligned}
$$

Some redundancies emerge for pairs of edges that are on complementary faces. On faces A and B for example are found respectively edges 1 and 2 which use the same conditions as in Eq.7. However, edges 1 and 3 for example have a special set of boundary conditions. The translation is given by

$$
\left\{\begin{array}{c}
\Delta x \\
\Delta y \\
\Delta z
\end{array}\right\}=\left\{\begin{array}{c}
2 b_{x} \\
2 b_{y} \\
0
\end{array}\right\}
$$

and hence

$$
\begin{aligned}
& \left(\left.u\right|_{y=b_{y}}-\left.u\right|_{y=-b_{y}}\right)=2 b_{x} \epsilon_{x}^{0}+2 b_{y} \gamma_{x y}^{0} \\
& \left(\left.v\right|_{y=b_{y}}-\left.v\right|_{y=-b_{y}}\right)=2 b_{y} \epsilon_{y}^{0} \\
& \left(\left.w\right|_{y=b_{y}}-\left.w\right|_{y=-b_{y}}\right)=0
\end{aligned}
$$

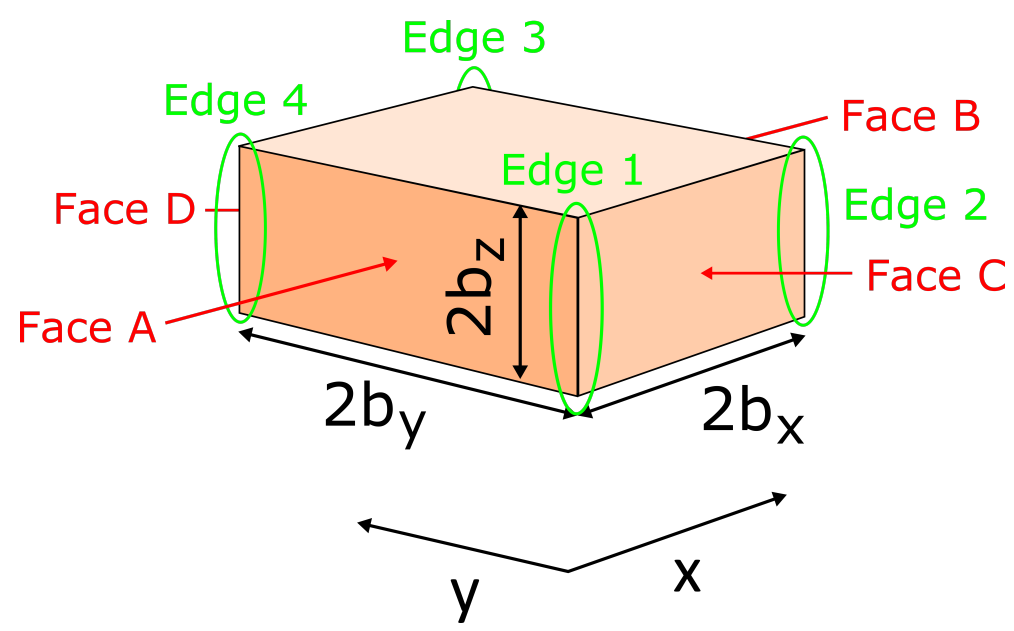

Figure 3: Unit cell's faces and edges numbering

It is of utmost importance that the mesh is similar for each pair of faces or edges.

\subsubsection{Effective material properties}

The macroscopic strains $\epsilon_{x}^{0}, \epsilon_{y}^{0}, \epsilon_{z}^{0}, \gamma_{x y}^{0}, \gamma_{y z}^{0}$ and $\gamma_{z x}^{0}$ appearing in the boundary conditions Eq.7-12 are physical entities and are called key DOFs [49], e.g. considered as six individual nodes, each having a single DOF. Six independent 
load cases $F_{x}, F_{y}, F_{z}, F_{x y}, F_{y z}$ and $F_{z x}$ are applied successively to the key DOFs, these concentrated forces are related to the macroscopic stresses $\sigma_{x}^{0}, \sigma_{y}^{0}, \sigma_{z}^{0}, \tau_{y z}^{0}, \tau_{z x}^{0}$ and $\tau_{x y}^{0}$ and the volume of the unit cell. This is performed in order to build the compliance matrix $[\mathbf{S}]$ of the macroscopic material by using the generalized Hooke's law: $\{\epsilon\}=[\mathbf{S}]\{\sigma\}$ (see Eq.13).

The material of the textile composite is considered orthotropic in its principal axes once homogenised, which makes the evaluation of the coefficients straightforward. Indeed, an orthotropic material is characterised by 9 engineering elastic constants only.

$$
\left\{\begin{array}{c}
\epsilon_{1} \\
\epsilon_{2} \\
\epsilon_{3} \\
\gamma_{23} \\
\gamma_{31} \\
\gamma_{12}
\end{array}\right\}=\left[\begin{array}{cccccc}
\frac{1}{E_{1}} & -\frac{v_{21}}{E_{2}} & -\frac{v_{31}}{E_{3}} & 0 & 0 & 0 \\
-\frac{v_{12}}{E_{1}} & \frac{1}{E_{2}} & -\frac{v_{32}}{E_{3}} & 0 & 0 & 0 \\
-\frac{v_{13}}{E_{1}} & -\frac{v_{23}}{E_{2}} & \frac{1}{E_{3}} & 0 & 0 & 0 \\
0 & 0 & 0 & \frac{1}{G_{23}} & 0 & 0 \\
0 & 0 & 0 & 0 & \frac{1}{G_{13}} & 0 \\
0 & 0 & 0 & 0 & 0 & \frac{1}{G_{12}}
\end{array}\right]\left\{\begin{array}{c}
\sigma_{1} \\
\sigma_{2} \\
\sigma_{3} \\
\tau_{23} \\
\tau_{31} \\
\tau_{12}
\end{array}\right\}
$$

For example, the first equation would be:

$$
\frac{1}{E_{1}} \sigma_{1}-\frac{v_{21}}{E_{2}} \sigma_{2}-\frac{v_{31}}{E_{3}} \sigma_{3}=\epsilon_{1}
$$

which gives in our case and when $F_{y}=F_{z}=F_{x y}=F_{y z}=F_{z x}=0$ :

$$
\frac{\sigma_{x}^{0}}{E_{x}^{0}}=\frac{F_{x}}{V_{t o t} E_{x}^{0}}=\epsilon_{x}^{0}
$$

The density for the macroscale model is calculated using the volume ratio of each material in the mesoscale model (weighted average formula), as follows

$$
\rho_{\text {macro }}=\frac{\rho_{f} V_{f}+\rho_{m} V_{m}}{V_{\text {tot }}}
$$

\subsection{System reduction using the Craig-Bampton Method}

The nodal DOFs of the unit cell are partitioned in the following way: boundary DOFs (themself partitioned as bottom, top, left, right, left-bottom corner, right-bottom corner, left-top corner, right-top corner) and internal DOFs (see Fig.4), which gives the following equation (subscript $b d$ stands for boundary):

$$
\mathbf{q}=\left\{\begin{array}{ll}
\mathbf{q}_{\text {bd }}^{\mathbf{T}} & \mathbf{q}_{\mathbf{I}}^{\mathbf{T}}
\end{array}\right\}^{T}=\left\{\begin{array}{lllllllll}
\mathbf{q}_{\mathbf{B}}^{\mathbf{T}} & \mathbf{q}_{\mathbf{T}}^{\mathbf{T}} & \mathbf{q}_{\mathbf{L}}^{\mathbf{T}} & \mathbf{q}_{\mathbf{R}}^{\mathbf{T}} & \mathbf{q}_{\mathbf{L B}}^{\mathbf{T}} & \mathbf{q}_{\mathbf{R B}}^{\mathbf{T}} & \mathbf{q}_{\mathbf{L T}}^{\mathbf{T}} & \mathbf{q}_{\mathbf{R T}}^{\mathbf{T}} & \mathbf{q}_{\mathbf{I}}^{\mathrm{T}}
\end{array}\right\}^{T}
$$




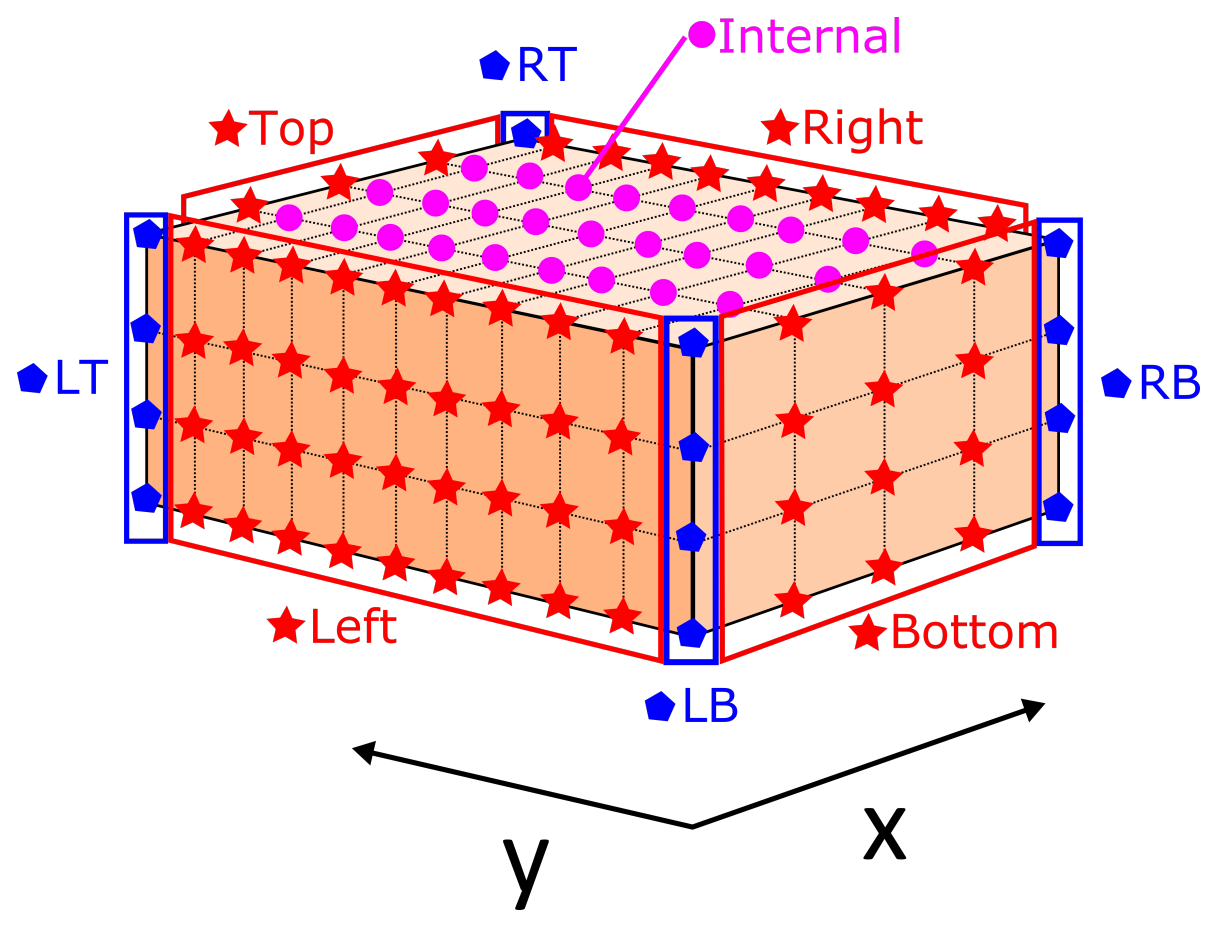

Figure 4: Partitioning of the degrees of freedom of a unit cell; '*' side nodes: bottom, top, left, right; 'diamond' corner nodes: LT (left-bottom), RB (right-bottom), LT (left-top), RT (right-top); 'o' internal nodes

The equation of motion of the unit cell is written as:

$$
\mathbb{M} \ddot{\mathbf{q}}(t)+\mathbb{C} \dot{\mathbf{q}}(t)+\mathbb{K} \mathbf{q}(t)=\mathbf{f}(t)
$$

Assuming time-harmonic behaviour leads to $\ddot{\mathbf{q}}=-\omega^{2} \mathbf{q}$ and considering no damping, Eq.18 can be rewritten as follows (Eq.19):

$$
\left[\mathbf{K}-\omega^{2} \mathbf{M}\right] \mathbf{q}=\mathbf{F}
$$

\subsubsection{Overview of the Craig-Bampton Method}

Component Mode Synthesis (CMS) are standard methods to reduce the complexity of structural dynamics models leading to reduced CPU time cost. The Craig-Bampton Method introduced in [50] is one of the CMS approaches. The boundary nodal DOFs are written as shown in Eq.17. For free wave propagation, no external force acts on the internal nodes of the structure. This leads to $\mathbf{f}_{\mathbf{I}}=\mathbf{0}$. The equation (Eq.19) of motion of the unit cell becomes:

$$
\left(\left[\begin{array}{cc}
\mathbf{K}_{\text {bdbd }} & \mathbf{K}_{\text {bdI }} \\
\mathbf{K}_{\text {Ibd }} & \mathbf{K}_{\mathbf{I I}}
\end{array}\right]-\omega^{2}\left[\begin{array}{cc}
\mathbf{M}_{\text {bdbd }} & \mathbf{M}_{\text {bdI }} \\
\mathbf{M}_{\mathbf{I b d}} & \mathbf{M}_{\mathbf{I I}}
\end{array}\right]\right)\left\{\begin{array}{c}
\mathbf{q}_{\mathbf{b d}} \\
\mathbf{q}_{\mathbf{I}}
\end{array}\right\}=\left\{\begin{array}{c}
\mathbf{f}_{\mathbf{b d}} \\
\mathbf{0}
\end{array}\right\}
$$


The key to this method is the reduction of the internal nodal DOFs: $\mathbf{q}_{\boldsymbol{\Phi}}$ is the reduced set of the physical internal DOFs $\mathbf{q}_{\mathbf{I}}$, whereas the boundary DOFs are kept as physical coordinates [50, 51]. In the Craig-Bampton CMS, a set of 'fixed boundary' modes, also called 'component' modes $\boldsymbol{\Phi}_{\mathbf{C}}$ are selected among a subset $\boldsymbol{\Phi}_{\mathbf{I}}$ of the local modes of the unit cell when the boundary DOFs are fixed and no force is acting on the internal nodes. $\boldsymbol{\Phi}_{\mathbf{I}}$ is obtained by solving the following eigenvalue problem:

$$
\left[\mathbf{K}_{\mathbf{I I}}-\omega^{2} \mathbf{M}_{\mathbf{I I}}\right] \boldsymbol{\Phi}_{\mathbf{I}}=\mathbf{0}
$$

$\boldsymbol{\Phi}_{\mathbf{b d}}$ represents the static boundary modes. It is called 'static' as it uses the system equations describing the unit cell behavior for a static analysis [50]:

$$
\mathbf{K q}=\mathbf{F}
$$

This gives

$$
\left[\begin{array}{cc}
\mathbf{K}_{\text {bdbd }} & \mathbf{K}_{\text {bdI }} \\
\mathbf{K}_{\text {Ibd }} & \mathbf{K}_{\text {II }}
\end{array}\right]\left\{\begin{array}{c}
\mathbf{q}_{\text {bd }} \\
\mathbf{q}_{\mathbf{I}}
\end{array}\right\}=\left\{\begin{array}{c}
\mathbf{f}_{\text {bd }} \\
\mathbf{0}
\end{array}\right\}
$$

From Eq.23, it can be written

$$
\mathbf{K}_{\text {Ibd }} \mathbf{q}_{\text {bd }}+\mathbf{K}_{\text {II }} \mathbf{q}_{\mathrm{I}}=\mathbf{0}
$$

or

$$
\mathbf{q}_{\mathrm{I}}=-\mathbf{K}_{\mathrm{II}}^{-1} \mathbf{K}_{\mathrm{Ibd}} \mathbf{q}_{\mathrm{bd}}=\boldsymbol{\Phi}_{\mathrm{bd}} \mathbf{q}_{\mathbf{b d}}
$$

Thus, the matrix of static boundary modes $\boldsymbol{\Phi}_{\mathbf{b d}}$ is written as follows $[6,18,19,20,50]$

$$
\Phi_{\text {bd }}=-K_{\text {II }}^{-1} K_{\text {Ibd }}
$$

The Craig-Bampton transformation matrix $\mathbf{B}$ can be determined as follows $[6,18,19,20,50]$ 


$$
\left\{\begin{array}{c}
\mathbf{q}_{\mathrm{bd}} \\
\mathbf{q}_{\mathrm{I}}
\end{array}\right\}=\left[\begin{array}{cc}
\mathbf{I} & \mathbf{0} \\
\boldsymbol{\Phi}_{\mathrm{bd}} & \boldsymbol{\Phi}_{\mathbf{C}}
\end{array}\right]\left\{\begin{array}{c}
\mathbf{q}_{\mathrm{bd}} \\
\mathbf{q}_{\Phi}
\end{array}\right\}=\mathbf{B}\left\{\begin{array}{c}
\mathbf{q}_{\mathrm{bd}} \\
\mathbf{q}_{\Phi}
\end{array}\right\}
$$

The $\mathbf{B}$ matrix is the link between the physical coordinates and the hybrid and reduced ones. The mass and stiffness matrix $\mathbf{M}$ and $\mathbf{K}$ can then be projected on this basis [6, 18, 19, 20, 50].

$$
\tilde{\mathbf{K}}=\mathbf{B}^{\mathrm{T}} \mathbf{K B}, \quad \tilde{\mathbf{M}}=\mathbf{B}^{\mathrm{T}} \mathbf{M B}
$$

This provides an efficient and reduced basis allowing for describing the response of the internal DOFs. To be later used in the CMS method.

\subsubsection{Component modes selection}

In the Craig-Bampton component modes selection method, the modal selection is based on the lower resonance frequencies of the clamped model. That is the reason for selecting modes into the frequency range $\left[0,3 f_{\max }\right]$, with $f_{\max }$ being the maximum frequency of interest for the wave dispersion analysis $[6,19,20]$.

\section{Overview of the WFE method}

According to the periodic structure theory (PST) developed for 2D structures by Langley in [52], when a free wave travels along a waveguide the displacement between two opposite boundary sides of a cell differs only by a propagation factor. This unit cell needs however to be meshed in a similar way for each of its opposite boundaries, so that continuity in displacements and forces equilibrium is respected [10], as shown in Fig.5.

For example, if the wave motion is along the $x$ direction, then $\mathbf{q}_{\mathbf{R}}=\lambda_{x} \mathbf{q}_{\mathbf{L}}$. And thus the size of the problem can be reduced as an unknown variable is removed. By using PST, the WFE method, which allows wave numbers to be found for all the propagating waves by modelling only a period of the structure with standard FE, has been developed.

For two dimensional periodic composite structures, the wave motion is in the $O x y$ plane. This gives

$$
\begin{aligned}
& \mathbf{q}_{\mathbf{R}}=\lambda_{x} \mathbf{q}_{\mathbf{L}} ; \quad \mathbf{q}_{\mathbf{T}}=\lambda_{y} \mathbf{q}_{\mathbf{B}} \\
& \mathbf{q}_{\mathbf{R B}}=\lambda_{x} \mathbf{q}_{\mathbf{L B}} ; \quad \mathbf{q}_{\mathbf{L T}}=\lambda_{y} \mathbf{q}_{\mathbf{L B}} ; \quad \mathbf{q}_{\mathbf{R T}}=\lambda_{x} \lambda_{y} \mathbf{q}_{\mathbf{L B}}
\end{aligned}
$$

And thus 


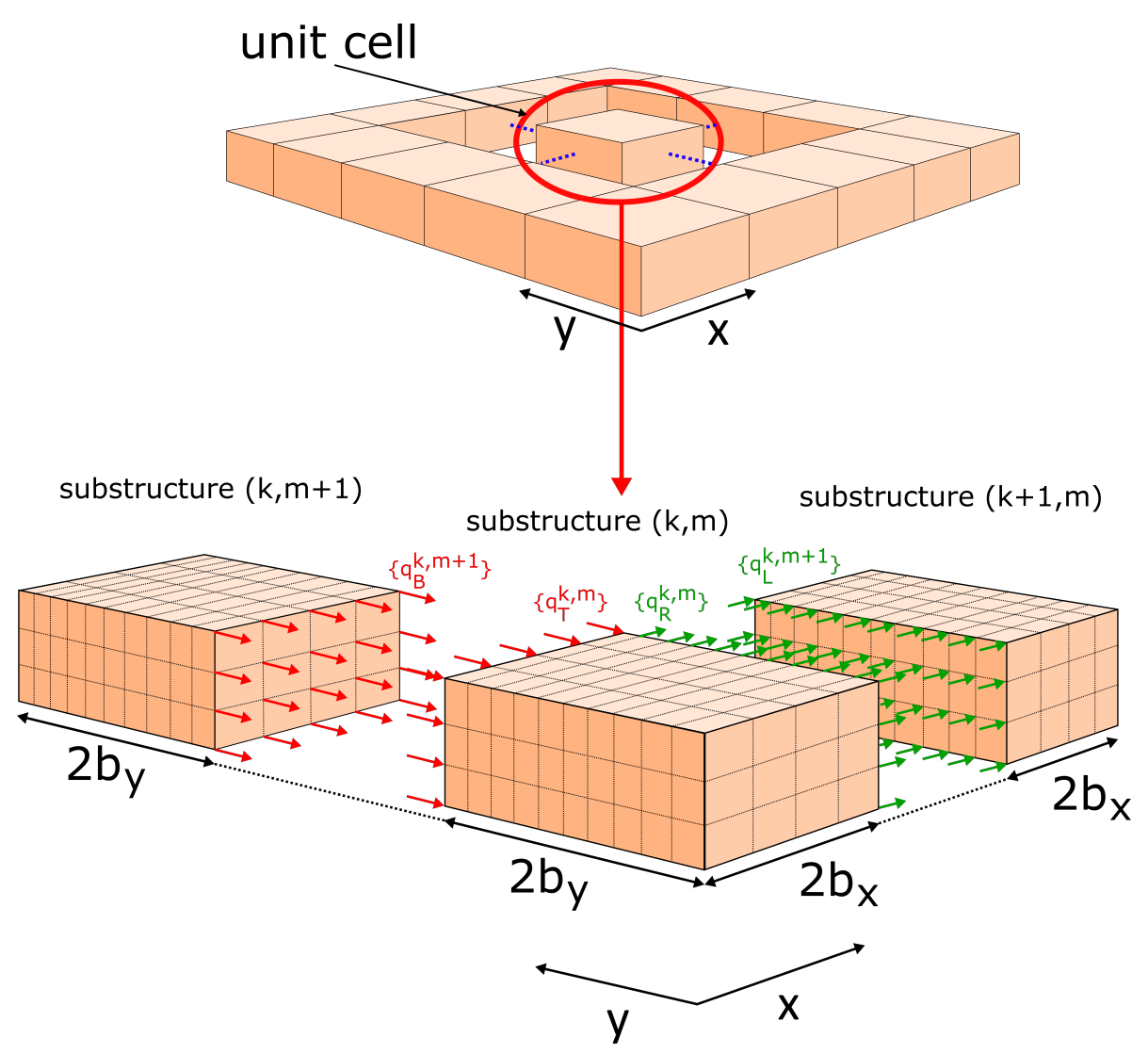

Figure 5: Decomposition of a periodic structure in an assembly of unit cells and presentation of the periodic structure theory [10]

$$
\left\{\begin{array}{c}
\mathbf{q}_{\mathbf{B}} \\
\mathbf{q}_{\mathbf{T}} \\
\mathbf{q}_{\mathbf{L}} \\
\mathbf{q}_{\mathbf{R}} \\
\mathbf{q}_{\mathbf{L B}} \\
\mathbf{q}_{\mathbf{R B}} \\
\mathbf{q}_{\mathbf{L T}} \\
\mathbf{q}_{\mathbf{R T}} \\
\mathbf{q}_{\mathbf{I}}
\end{array}\right\}=\left[\begin{array}{cccc}
\mathbf{I} & \mathbf{0} & \mathbf{0} & \mathbf{0} \\
\mathbf{I} \lambda_{y} & \mathbf{0} & \mathbf{0} & \mathbf{0} \\
\mathbf{0} & \mathbf{I} & \mathbf{0} & \mathbf{0} \\
\mathbf{0} & \mathbf{I} \lambda_{x} & \mathbf{0} & \mathbf{0} \\
\mathbf{0} & \mathbf{0} & \mathbf{I} & \mathbf{0} \\
\mathbf{0} & \mathbf{0} & \mathbf{I} \lambda_{x} & \mathbf{0} \\
\mathbf{0} & \mathbf{0} & \mathbf{I} \lambda_{y} & \mathbf{0} \\
\mathbf{0} & \mathbf{0} & \mathbf{I} \lambda_{x} \lambda_{y} & \mathbf{0} \\
\mathbf{0} & \mathbf{0} & \mathbf{0} & \mathbf{I}
\end{array}\right]\left\{\begin{array}{c}
\mathbf{q}_{\mathbf{B}} \\
\mathbf{q}_{\mathbf{L}} \\
\mathbf{q}_{\mathbf{L B}} \\
\mathbf{q}_{\mathbf{I}}
\end{array}\right\}=\boldsymbol{\Lambda}_{\mathbf{R}}\left(\lambda_{x}, \lambda_{y}\right)\left\{\begin{array}{c}
\mathbf{q}_{\mathbf{B}} \\
\mathbf{q}_{\mathbf{L}} \\
\mathbf{q}_{\mathbf{L B}} \\
\mathbf{q}_{\mathbf{I}}
\end{array}\right\}
$$

Equilibrium at the right top corner nodes gives: 


$$
\boldsymbol{\Lambda}_{\mathbf{L}}\left(\lambda_{x}, \lambda_{y}\right)\left\{\begin{array}{c}
\mathbf{f}_{\mathbf{b d}} \\
\mathbf{0}
\end{array}\right\}=\mathbf{0}
$$

with $\boldsymbol{\Lambda}_{\mathbf{L}}\left(\lambda_{x}, \lambda_{y}\right)$ the conjugate transpose of $\boldsymbol{\Lambda}_{\mathbf{R}}\left(\lambda_{x}, \lambda_{y}\right)$.

The following eigenvalue problem appears

$$
\boldsymbol{\Lambda}_{\mathbf{L}}\left(\mathbf{K}-\omega^{2} \mathbf{M}\right) \boldsymbol{\Lambda}_{\mathbf{R}}\left\{\begin{array}{c}
\mathbf{q}_{\mathbf{B}} \\
\mathbf{q}_{\mathbf{L}} \\
\mathbf{q}_{\mathbf{L B}} \\
\mathbf{q}_{\mathbf{I}}
\end{array}\right\}=0
$$

Combining the CMS reduction method Eq.27 with the periodicity relation Eq.30, we obtain

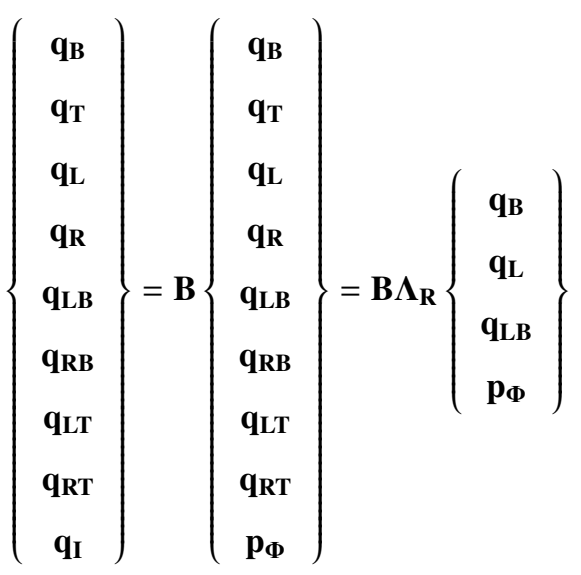

The eigenvalue problem can be solved with a given $\left(k_{x}, k_{y}\right)$ formulation [23] $\left(k_{x}\right.$ and $k_{y}$ being respectively the wavenumber in the $\mathrm{x}$ and $\mathrm{y}$ direction). Both $k_{x}$ and $k_{y}$ are taken in the range $\left[0, \frac{\pi}{\Delta_{x}}\right]$, respectively $\left[0, \frac{\pi}{\Delta_{y}}\right] \cdot \lambda=e^{-i k \Delta}$ is a periodic function, for that reason, the results are $\pi$ periodic and need to be post-processed to sort the dispersion curves. The Modal Assurance Criterion is used for that purpose. In Fig.6, the dispersion curves of the first modes of a model before and after post-processing are displayed.

\section{Numerical investigation of a $2 \mathrm{D}$ weave fabric}

As a first example, a 2D weave fabric composite is presented. In this section, dispersion curves for the modelled textile composites are presented. Even though the WFE calculations are performed in 2D, the results will be displayed in the $\Theta=0^{\circ}$ direction for the sake of clarity (except if specified otherwise). A unit cell is extracted from the fabric as shown in Fig.2, its dimensions are 2x2x0.2 mm. 


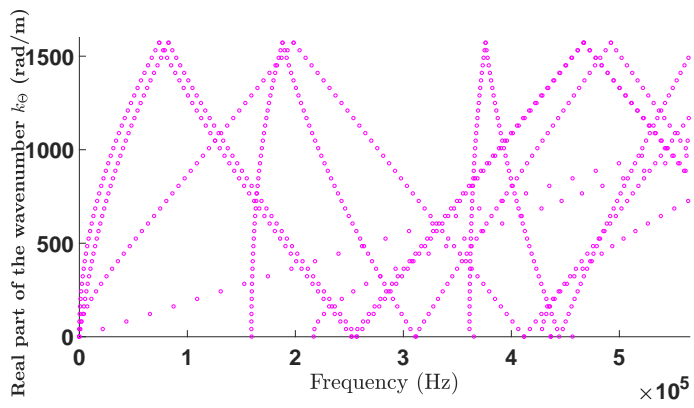

(a) Before sorting

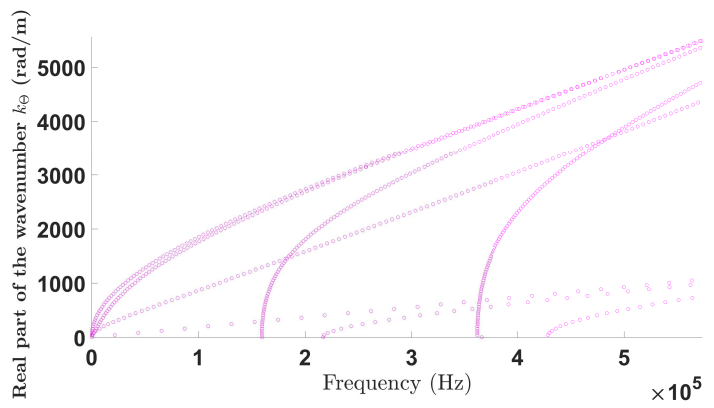

(b) After sorting

Figure 6: Solving the eigenvalue problem gives $\pi$ periodic results that need to be sorted, using the MAC criterion; (a) on the left figure, the dispersion curves (real part of the wavenumber against frequency) are displayed before post-processing; (b) on the right, the curves have been post-processed using the MAC criterion

\subsection{Mesoscale description}

\subsubsection{Mechanical properties}

The mechanical properties of the materials in the mesoscale textile composite are chosen as shown in Tables.1-2.

\begin{tabular}{|c|c|c|c|}
\hline Matrix & $\mathrm{E}\left(\mathrm{N} / \mathrm{m}^{2}\right)$ & $v$ & $\rho\left(\mathrm{kg} / \mathrm{m}^{3}\right)$ \\
\hline & $3 \times 10^{9}$ & 0.2 & 1600 \\
\hline
\end{tabular}

Table 1: Engineering constants of the isotropic matrix material for the 2D weave textile composite

\begin{tabular}{|c|c|c|c|c|c|c|}
\hline Yarn & $E_{1}\left(\mathrm{~N} / \mathrm{m}^{2}\right)$ & $E_{2}\left(\mathrm{~N} / \mathrm{m}^{2}\right)$ & $E_{3}\left(\mathrm{~N} / \mathrm{m}^{2}\right)$ & $v_{12}$ & $v_{13}$ & $v_{23}$ \\
\hline & $2 \times 10^{11}$ & $1 \times 10^{10}$ & $1 \times 10^{10}$ & 0.3 & 0.4 & 0.4 \\
\hline \hline & $G_{12}\left(\mathrm{~N} / \mathrm{m}^{2}\right)$ & $G_{13}\left(\mathrm{~N} / \mathrm{m}^{2}\right)$ & $G_{23}\left(\mathrm{~N} / \mathrm{m}^{2}\right)$ & $\rho\left(\mathrm{kg} / \mathrm{m}^{3}\right)$ & & \\
\hline & $5 \times 10^{9}$ & $5 \times 10^{9}$ & $5 \times 10^{9}$ & 4600 & & \\
\hline
\end{tabular}

Table 2: Engineering constants of the orthotropic yarn material used for the 2D weave fabric

\subsubsection{Mesh convergence study}

In this paragraph, four mesoscale FE models of a unique 2D weave textile are compared (four different meshes for an identical weave geometry). On the top left figure in Fig.7 is presented the geometrical model used as a reference. The four FE models are gradually refined, starting with a coarse anisotropic model containing 500 elements (10x10x5). The second model contains 2890 elements (17x17x10), the third contains 6250 elements $(25 \times 25 \times 10)$ while the last one is build up of 13500 elements (30x30x15). 


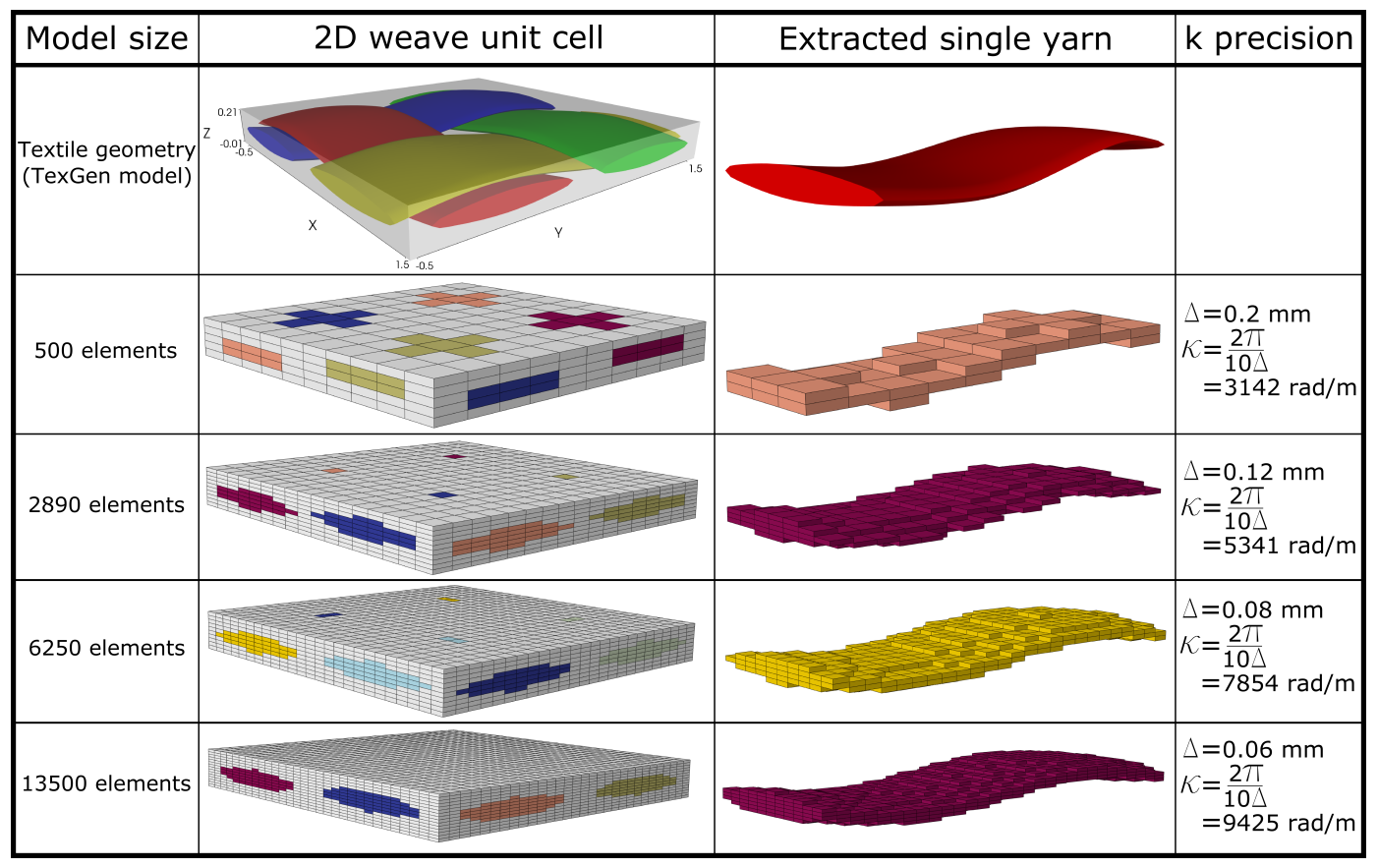

Figure 7: Meshed 2D mesoscale textile models; topological model provided by Texgen shown on top, multiscale models described by an increasing number of elements in the following rows

6-10 elements per wavelength have to be used as a minimum, as a rule of thumb, in order to obtain accurate results. This is how the 'k precision' is calculated in Fig.7.

A convergence study is performed on those four models in order to use a low number of degrees of freedom while having results as accurate as possible. The relative error is calculated for the three first modes of each model, using the finer model as a reference. In Fig.8-10 are displayed the first three modes for each model in order to allow for comparison between the different meshes. It seems that a convergence can be observed, and even though the 500 elements model is coarse, the maximum relative difference with the finest model (13500 elements) is only 6.5\%, and the shape of the dispersion curve for each modes are similar.

Fig.11 shows the relative error as previously described. It can be observed that the mean relative difference goes below $1 \%$ from the third model composed of 6250 elements, the convergence is confirmed. This model will be used for the rest of the study.

For the first model composed of 500 elements, the calculation time to obtain the dispersion relations is around 4 minutes, the second model composed of 2890 elements has a calculation time of around 20 minutes and finally, the chosen model composed of 6250 elements has a computation time of around 50 minutes.

It has to be stressed that all models successfully predict the stop-band behaviour of the textile composite within 


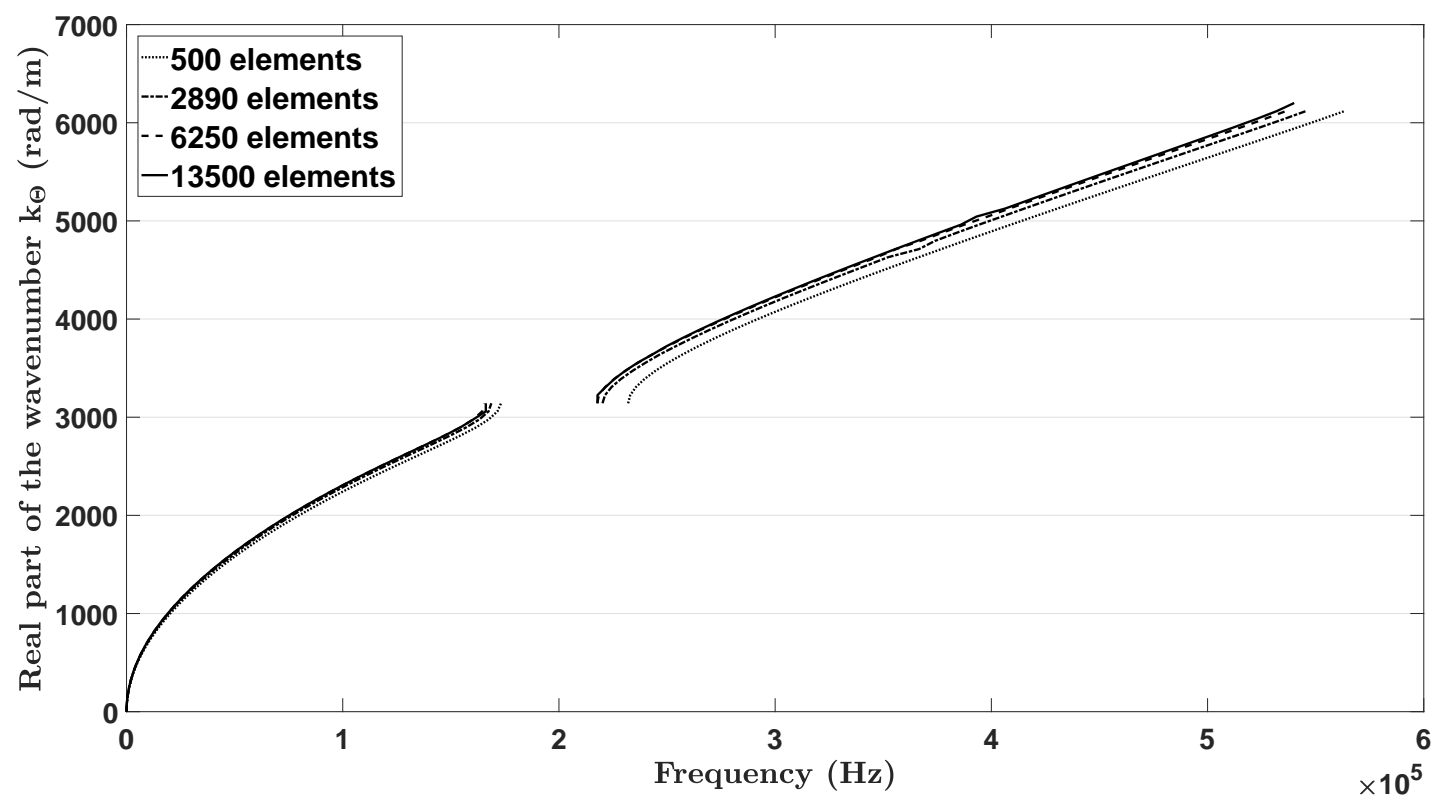

Figure 8: Dispersion curve of the first anti-symmetric flexural mode; '..' results for the model composed of 500 elements, '-'. results for the model composed of 2890 elements, '- -' results for the model composed of 6250 elements and '-' results for the model composed of 13500

specific frequency ranges. It is reminded that wave propagation is forbidden within these ranges, therefore guided wave inspection with a corresponding wave type is not possible.

\subsubsection{Influence of the CMS reduction method on the results}

In this paragraph, the impact of the CMS reduction method on the results is studied: two modelling methods are compared, one using both the WFE and CMS methods, another using only the WFE method for reduction of the system size. The results are displayed in Fig.12. It can be seen that the CMS reduction method shows almost no error for this range of frequency (mean relative difference of $0.06 \%$ for the flexural mode, $0.27 \%$ for the shear mode and $0.13 \%$ for the extensional mode).

\subsection{Macroscale homogenisation}

The macroscopic material properties are found using the mesoscopic material properties given in Tab. 1 and 2 through the methodology described in section 2.2.

The computed homogenised properties are shown in Tab.3.

The Texgen software directly provides the volume fraction of the investigated textiles. For example, in our case, the fibre volume fraction for whole yarns is 0.54 of the full volume. 


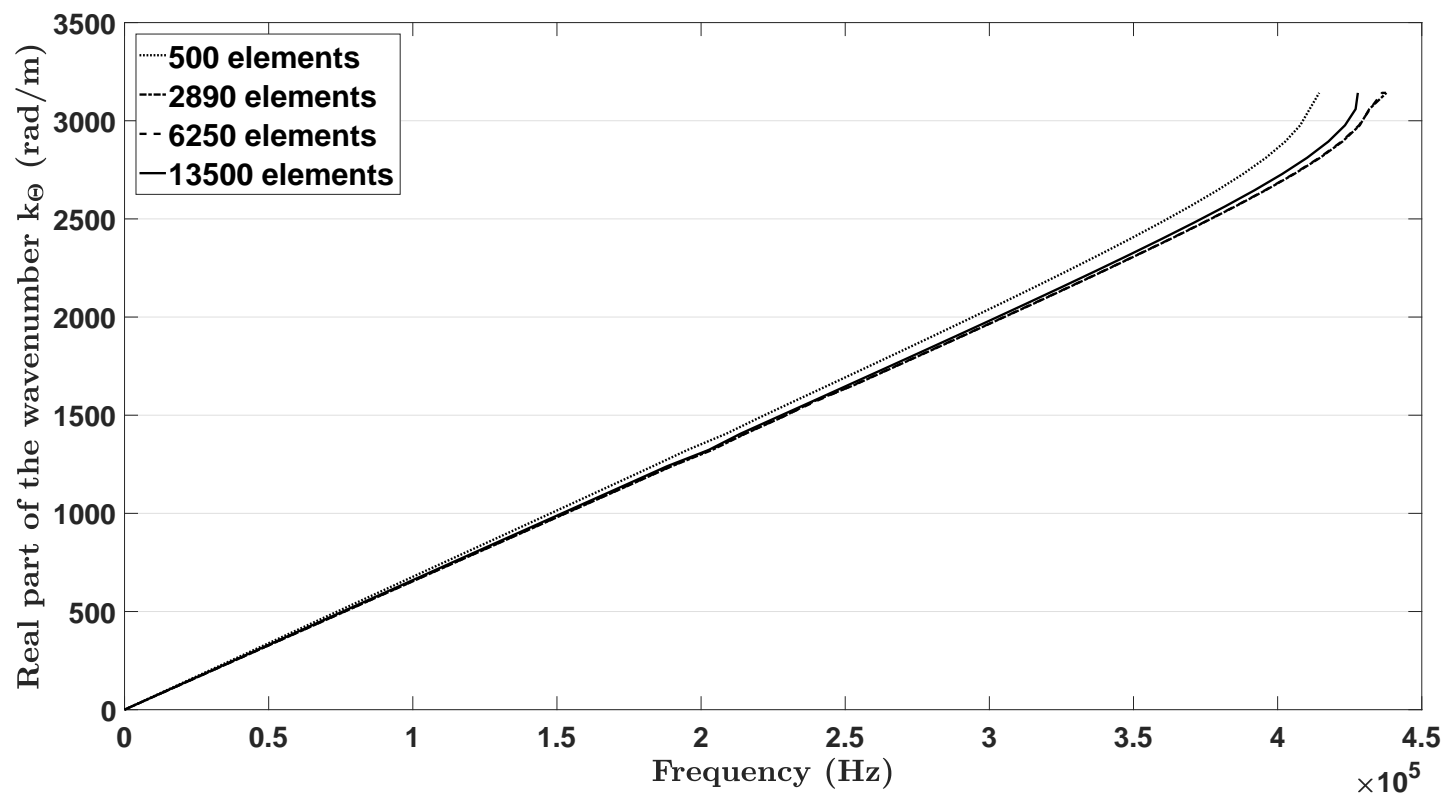

Figure 9: Dispersion curve of the SH (shear-horizontal) mode; '..' results for the model composed of 500 elements, '-.' results for the model composed of 2890 elements, '- -' results for the model composed of 6250 elements and '-' results for the model composed of 13500

\begin{tabular}{|c|c|c|c|c|c|}
\hline$E_{1}\left(\mathrm{~N} / \mathrm{m}^{2}\right)$ & $E_{2}\left(\mathrm{~N} / \mathrm{m}^{2}\right)$ & $E_{3}\left(\mathrm{~N} / \mathrm{m}^{2}\right)$ & $G_{12}\left(\mathrm{~N} / \mathrm{m}^{2}\right)$ & $G_{13}\left(\mathrm{~N} / \mathrm{m}^{2}\right)$ & $G_{23}\left(\mathrm{~N} / \mathrm{m}^{2}\right)$ \\
\hline $3.73 \times 10^{10}$ & $3.73 \times 10^{10}$ & $6.05 \times 10^{9}$ & $2.54 \times 10^{9}$ & $2.43 \times 10^{9}$ & $2.43 \times 10^{9}$ \\
\hline \hline$v_{12}$ & $v_{13}$ & $v_{21}$ & $v_{23}$ & $v_{31}$ & $v_{32}$ \\
\hline 0.136 & 0.362 & 0.136 & 0.362 & 0.059 & 0.059 \\
\hline
\end{tabular}

Table 3: Orthotropic homogenised engineering constants of the macroscale 2D weave textile composite

The density is:

$$
\begin{aligned}
\rho_{\text {macro }} & =\rho_{f} V_{f}^{\text {frac }}+\rho_{m}\left(1-V_{f}^{\text {frac }}\right) \\
& =4600 \times 0.54+1600 \times(1-0.54)=3220 \mathrm{~kg} \cdot \mathrm{m}^{3}
\end{aligned}
$$

These mechanical properties are used for creating the macroscale model; the aim being to compare this model to the 6250 elements mesoscale model. The modelled element needs to have the same dimensions as the ones of the mesoscale model. The WFE method is then performed on a model composed of one element in both the $x$ and $y$ directions and of ten elements in the $z$ direction $(1 \times 1 \times 10)$. The dispersion curves for the first three modes of this model are displayed in Fig.13-15, as well as the modes of the 6250 elements mesoscale model. 


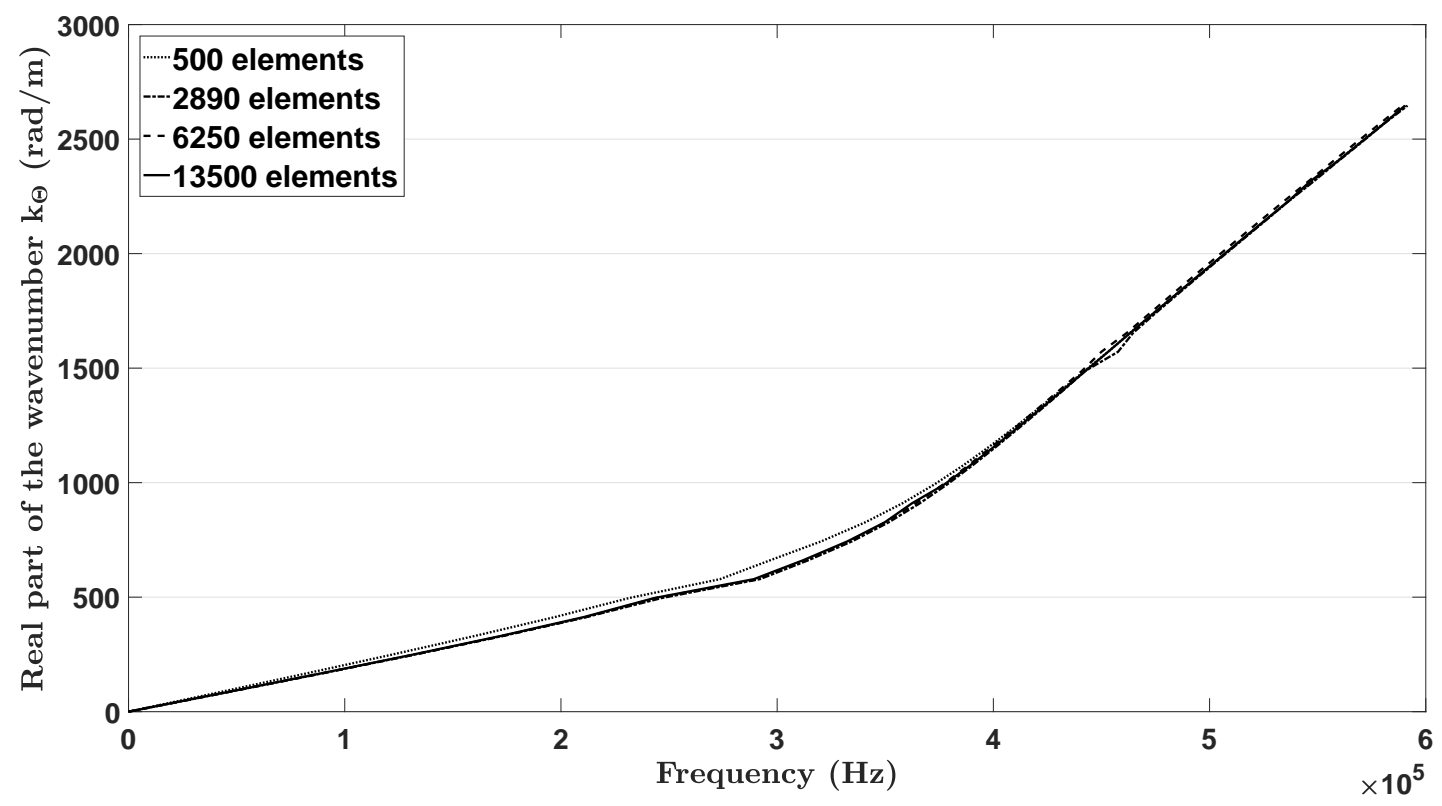

Figure 10: Dispersion curve of the pressure mode; '..' results for the model composed of 500 elements, '-.' results for the model composed of 2890 elements, '- -' results for the model composed of 6250 elements and '-' results for the model composed of 13500

In Fig.13-15, we notice some important differences between the curves from the meso- and macroscale models. The mean relative frequency difference for the flexural mode (Fig.13) is of $31.5 \%$, and the dispersion curve present a stop-band when modelled at a mesoscale. In the case of the shear mode (Fig.14), it can be observed that both models show very similar results. The relative difference is of $2.8 \%$ in that case and the results mainly differ by the shape of the curve from a wavenumber of $2500 \mathrm{rad} / \mathrm{m}$. Indeed, from a wavenumber of $3000 \mathrm{rad} / \mathrm{m}$, the dispersion curve of the shear mode of the mesoscale model present a stop-band. At last, the mean relative difference for the pressure mode (Fig. 15 ) is of $14.7 \%$ in the wavenumber range $[0,600] \mathrm{rad} / \mathrm{m}$, but is then increasing to almost reach $50 \%$ of relative difference. As the global density and the global mechanical properties of both models are the same, the only factor that could be affecting the dispersion is the internal geometry of the unit cell.

It can be noted that stop-bands occur (Fig.13-14) when the wavenumber reaches $k_{x}=\frac{2 \pi}{\Delta_{x}}$, which means when the wavelength is of the size of a periodicity of the structure. In Fig.13, the stop-band is located in the frequency range $[1.7 \mathrm{e} 5,2.2 \mathrm{e} 5] \mathrm{Hz}$ (grayed area). This means that no flexural wave will propagate at this frequency range.

\subsection{Numerical validation using guided waves in a $2 D$ weave structure fully modelled through $F E s$}

In order to validate the method used in this paper, a comparison with a structure fully modelled through FEs will be drawn. The phase velocity and group velocity of a longitudinal wave will be investigated versus frequency in a beam structure made of the $2 \mathrm{D}$ weave fabric presented in section 4.1. A commercial FE software is used to obtain the 


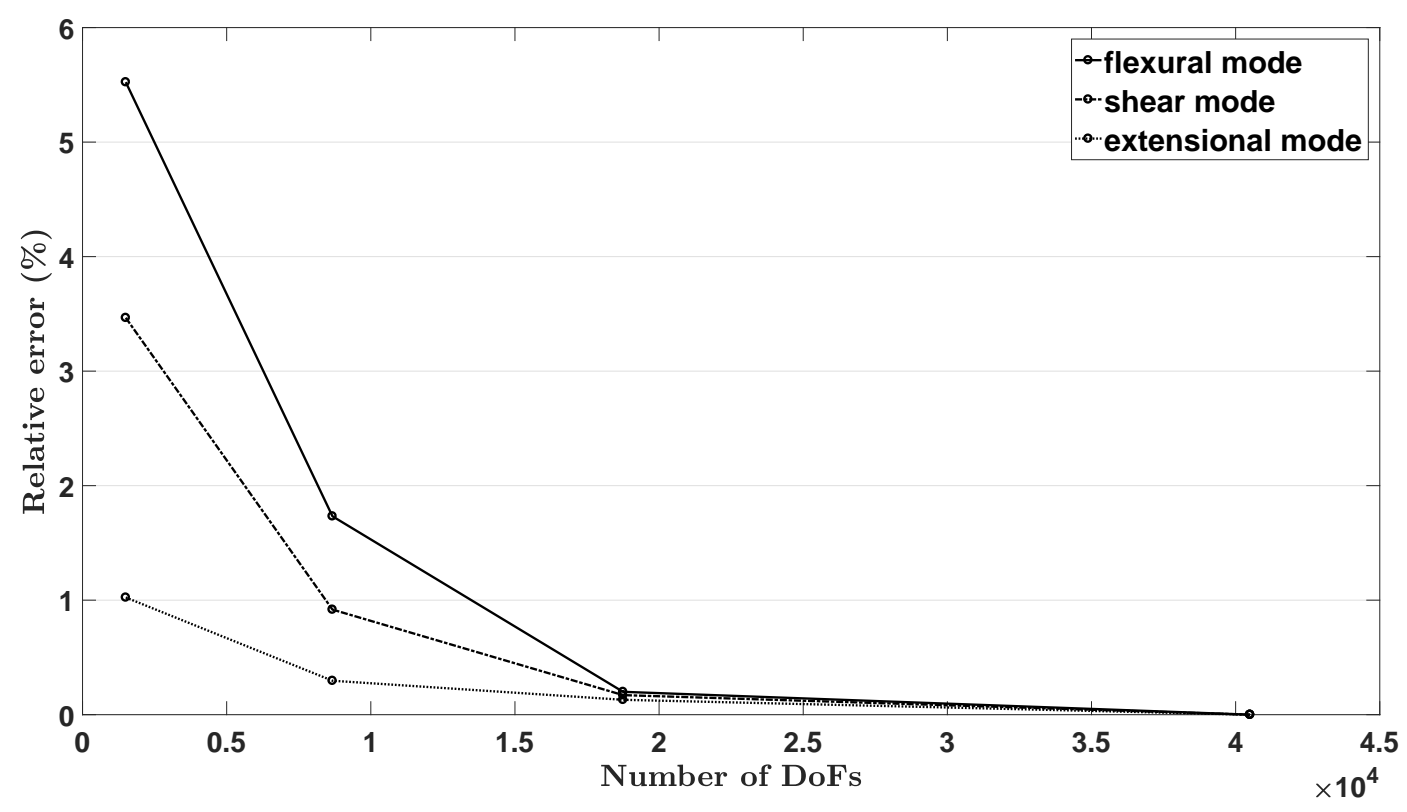

Figure 11: Mean relative difference calculated for the dispersion curve of the first three modes of each model, displayed as a function of the number of degrees of freedom; '- o' relative error for the flexural mode, '-.o' relative error for the shear mode, '..o' relative error for the extensional mode

results presented in this section.

\subsubsection{Theoretical model description}

A beam made of 2D weave fabric is modelled. Its width is of $2 \mathrm{~mm}$, while its thickness is of $0.2 \mathrm{~mm}$. These dimensions are used so that the model is composed of only one unit cell in its width and thickness while an extensive number of unit cells are used in its length. This way the propagating waves reflecting at the far end do not affect the results.

Longitudinal waves are chosen for the study as they are straightforward to induce to a numerical beam model. A force envelope is applied on one end side of the beam in the direction of the beam length. The magnitude of the load is variable over time so that the signal carries a narrow band frequency, and has a short time pulse. To avoid the coupling of various modes, displacement in the width and thickness directions are set as null independently of time. This is also allowing for shorter computation time.

The signal is composed of a signal carrying the frequency of interest, mixed with a Hann window function (see Fig.16). The carrier signal is periodic sinusoidal and composed of eleven cycles. It is defined as follows (Eq.35):

$$
v(t)=\sin \left(2 \pi f_{c} t\right)
$$




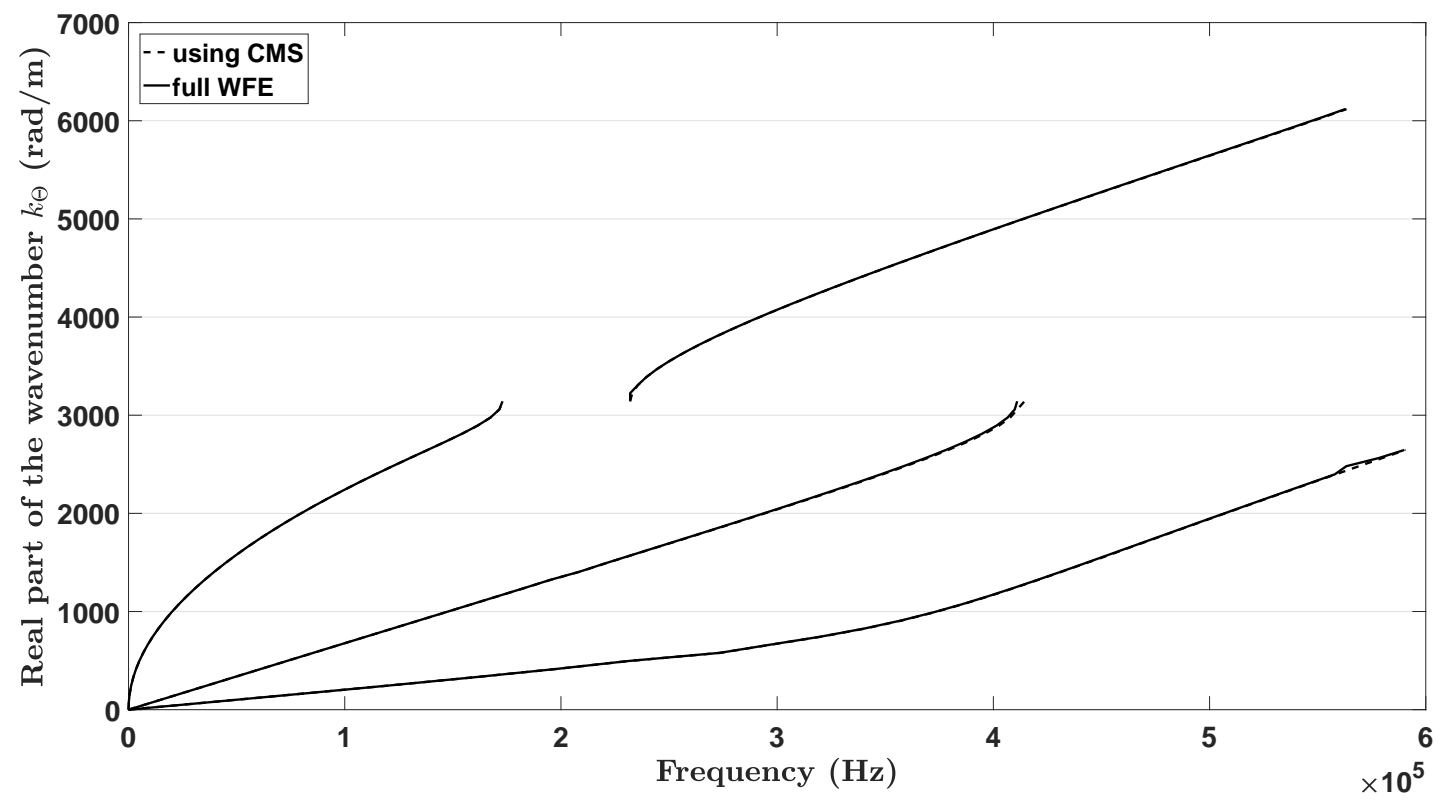

Figure 12: Dispersion curves of the flexural, shear and extensional modes for a same model but different reduction methods; '- -' using both the CMS and WFE methods and '-' using only the WFE method)

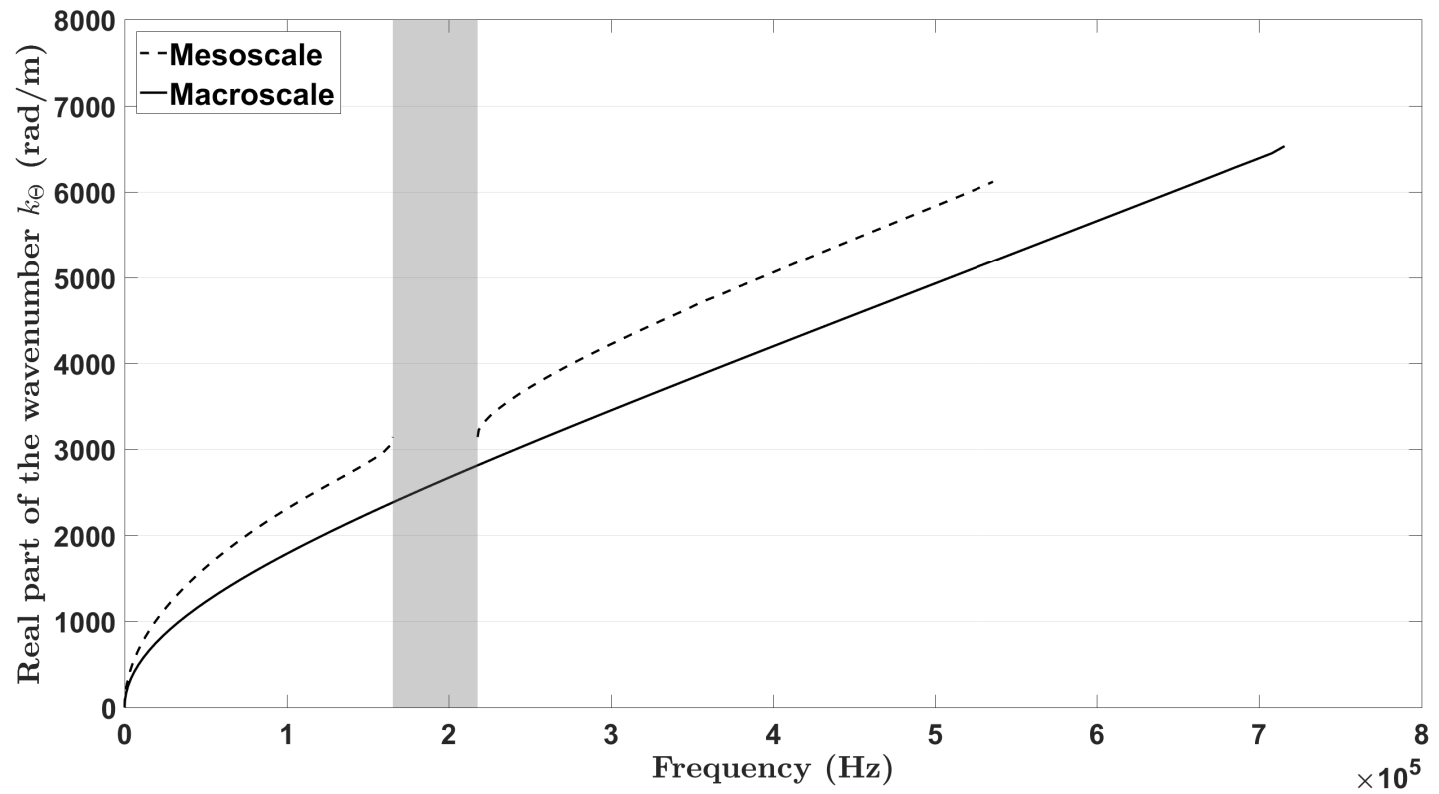

Figure 13: Dispersion curve of the flexural mode of the mesoscale model and the macroscale model; presenting a stop-band (grayed area) for the the mesoscale model; '- -' mesoscale model and '-' macroscale model 


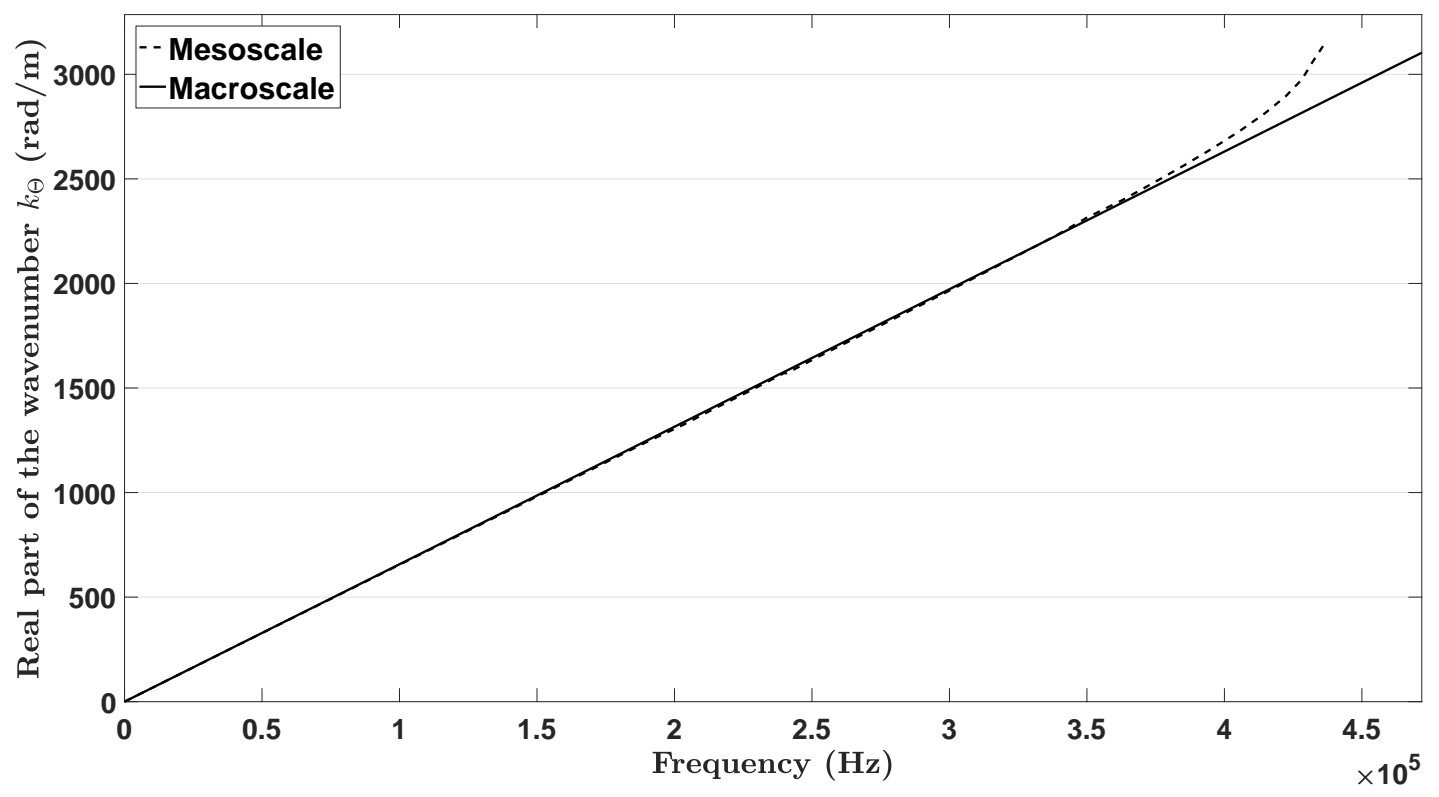

Figure 14: Dispersion curve of the shear mode of the mesoscale model and the macroscale model; '- -' mesoscale model and '-' macroscale model

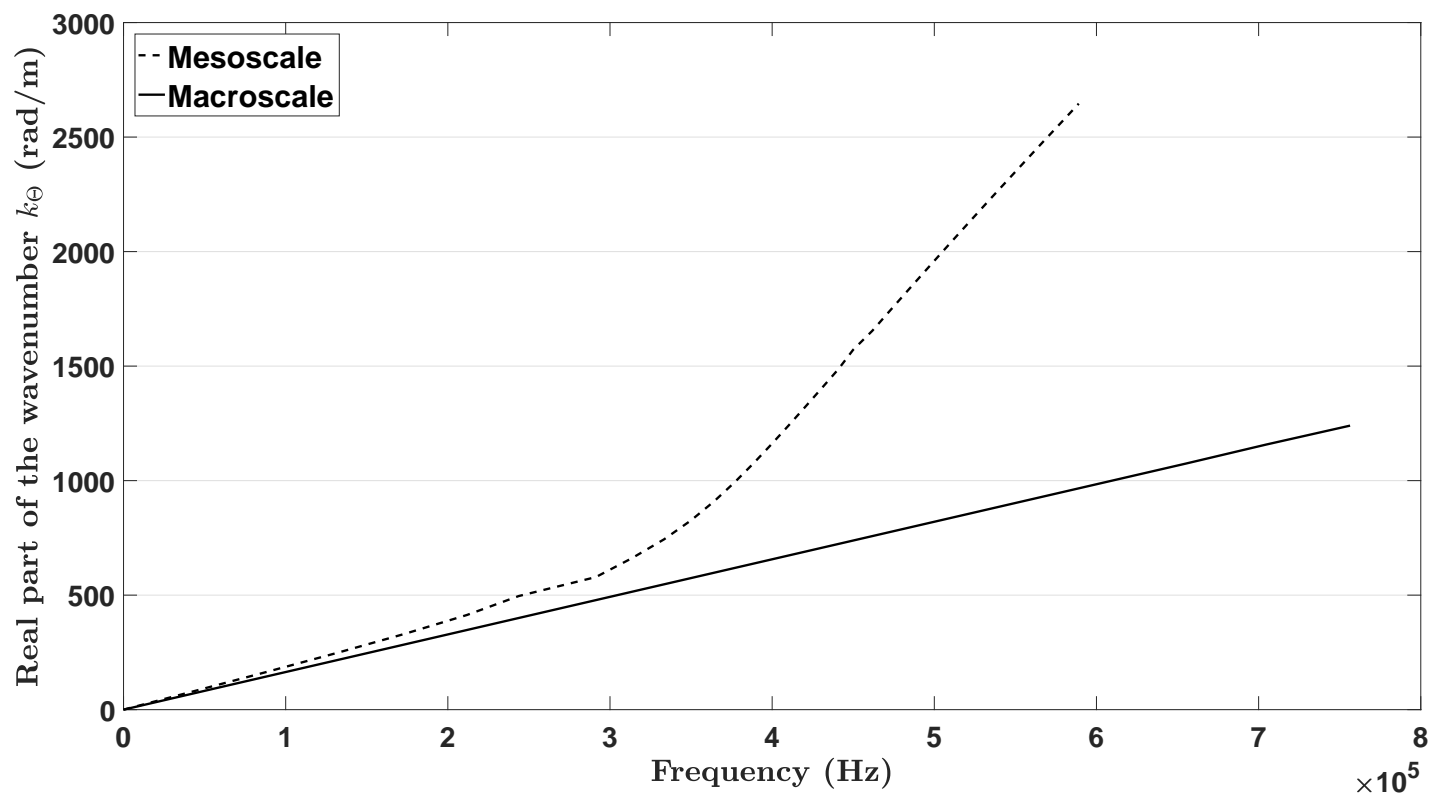

Figure 15: Dispersion curve of the extensional mode of the mesoscale model and the macroscale model; '- -' mesoscale model and '-' macroscale model 
$v(t)$ is the amplitude of the signal, $f_{c}$ the frequency of interest and $t$ the time. $t$ varies from 0 to $\frac{11}{f_{c}}$.

The Hann window function is a discrete function and is defined as follows (Eq.36):

$$
w(n)=\sin ^{2}\left(\frac{\pi n}{N-1}\right)
$$

$w(n)$ is the amplitude, $N$ the size of the sample, this must correspond with the number of discrete values used to define the carrier signal.

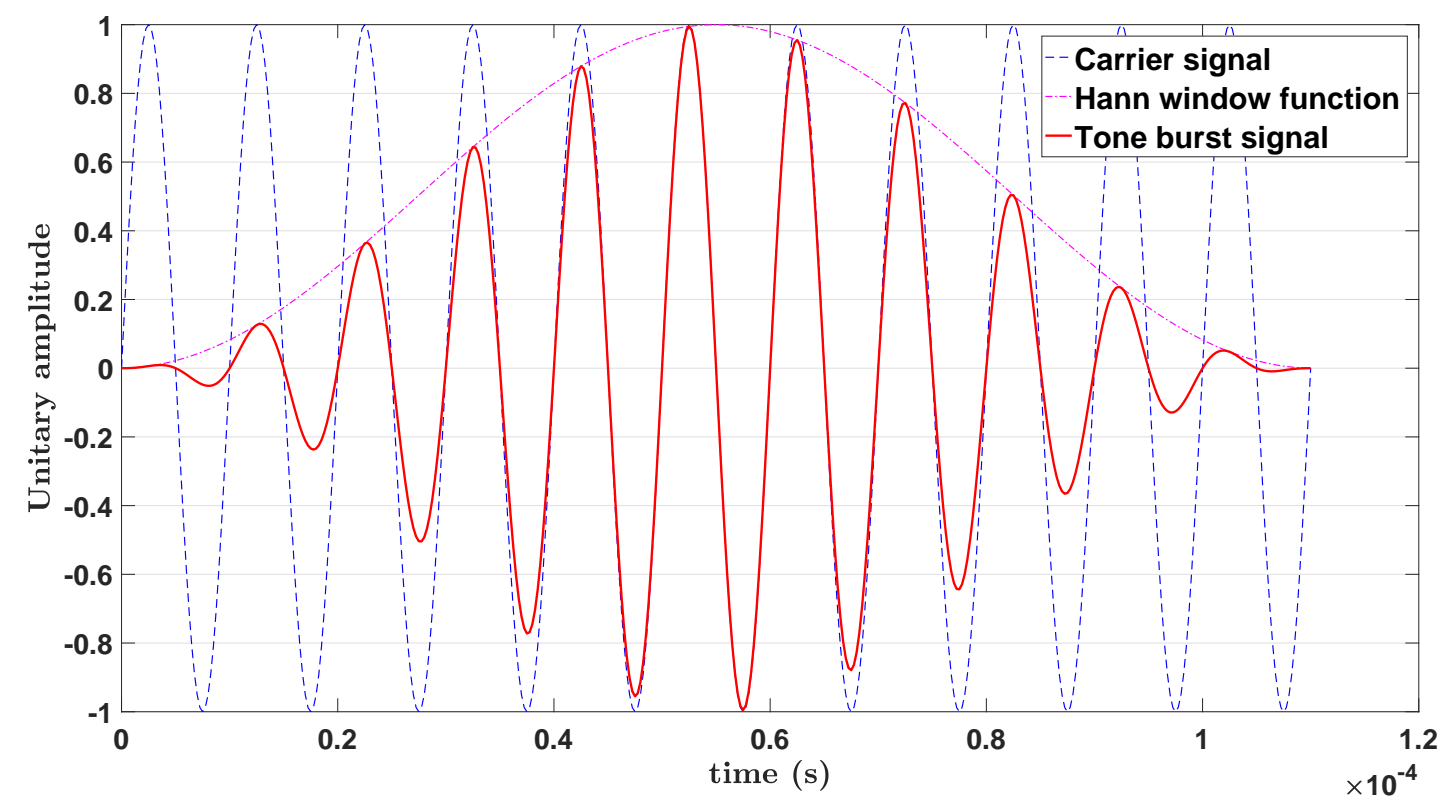

Figure 16: The two components of the signal are the '- -' carrier signal and '-.' the Hann window function. The carrier signal is carrying a frequency of $1 \mathrm{e} 5 \mathrm{~Hz}$. Combining these two components gives '-' the resulting signal

The displacement in the length direction is measured over time at different length positions of the beam. Using these displacement amplitude over time data, the phase velocity and group velocity of the wave packet can be calculated. A parametric study is conducted, using signals carrying wave packets of different frequencies, in order to obtain the sought dispersion relations.

\subsubsection{Finite Element model}

Data is captured at three different distances along the length of the beam on the upper surface of the model and again at three different distances on the lower surface of the beam, to verify this has no impact on the results. For an 
accurate comparison, the model mesh size is the same for the two compared methods (full FE and WFE/CMS). The length of the modelled beam varies according to the investigated frequency (around 10 wavelengths). The results are computed for a beam made of 2D weave fabric modelled at a mesoscopic scale (as presented in section 4.1, tables 1-2) as well as at a macroscopic scale (see section 4.2, table 3).

Results.

The WFE/CMS method shown in this paper allows for computing the wavenumber (as displayed in Fig.18), the phase velocity (as shown in Fig.19) and the group velocity (as shown in Fig.20) over frequency. This method is used for the described beam model to obtain the dispersion relations as shown in Fig.18-22.

The full FE model allows for computing the phase velocity of the propagating wave as well as the group velocity and it is shown along with the results from the WFE/CMS method in Fig.21-22. The phase velocity is calculated by considering the velocity at which an individual peak propagates (taking here the central crest of the signal) [53]. The envelope of the captured signal is calculated using the Hilbert transform (see Fig.17), the group velocity is the velocity at which the envelope propagates (the crest of the envelope is considered as the reference point) [54].

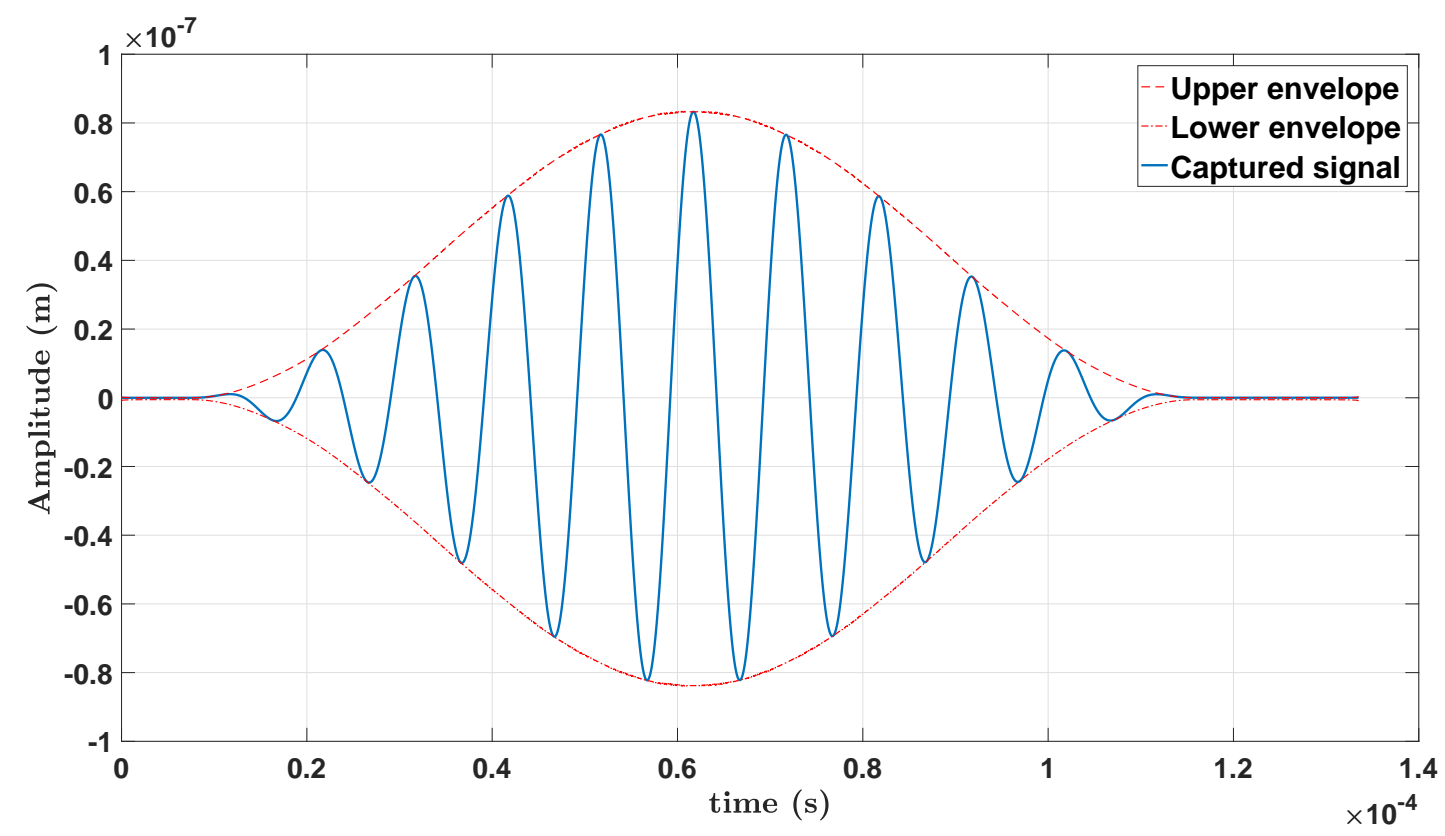

Figure 17: Captured signal at $x=26 \mathrm{~mm}$, for an input signal of $1 \mathrm{e} 5 \mathrm{~Hz}$, in the beam considered at a macroscopic scale, with its upper and lower envelopes calculated using the Hilbert transform; '- -' upper envelope, '-.' lower envelope and '-' captured signal

Once again, important differences exist between the dispersion relations of the model when considered at a mesoor macroscale. For example, in Fig.18, when considering the first mode, the relative wavenumber difference for a given frequency grows with the frequency. At $700 \mathrm{kHz}$, the difference is of almost 10\%. Another great difference is 


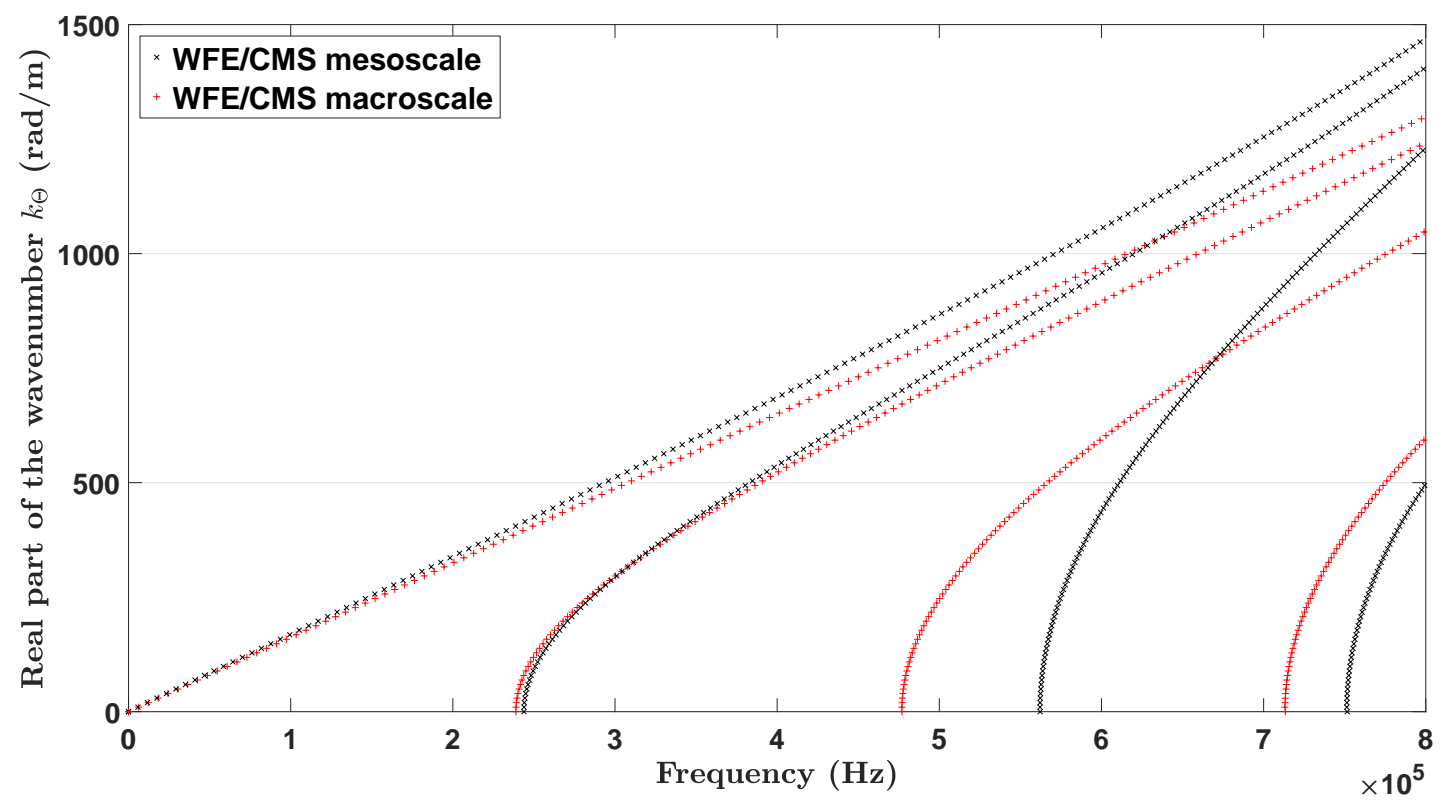

Figure 18: Dispersion curves of the mesoscale model and macroscale model, obtained through the WFE/CMS method; 'x' mesoscale and '+' macroscale

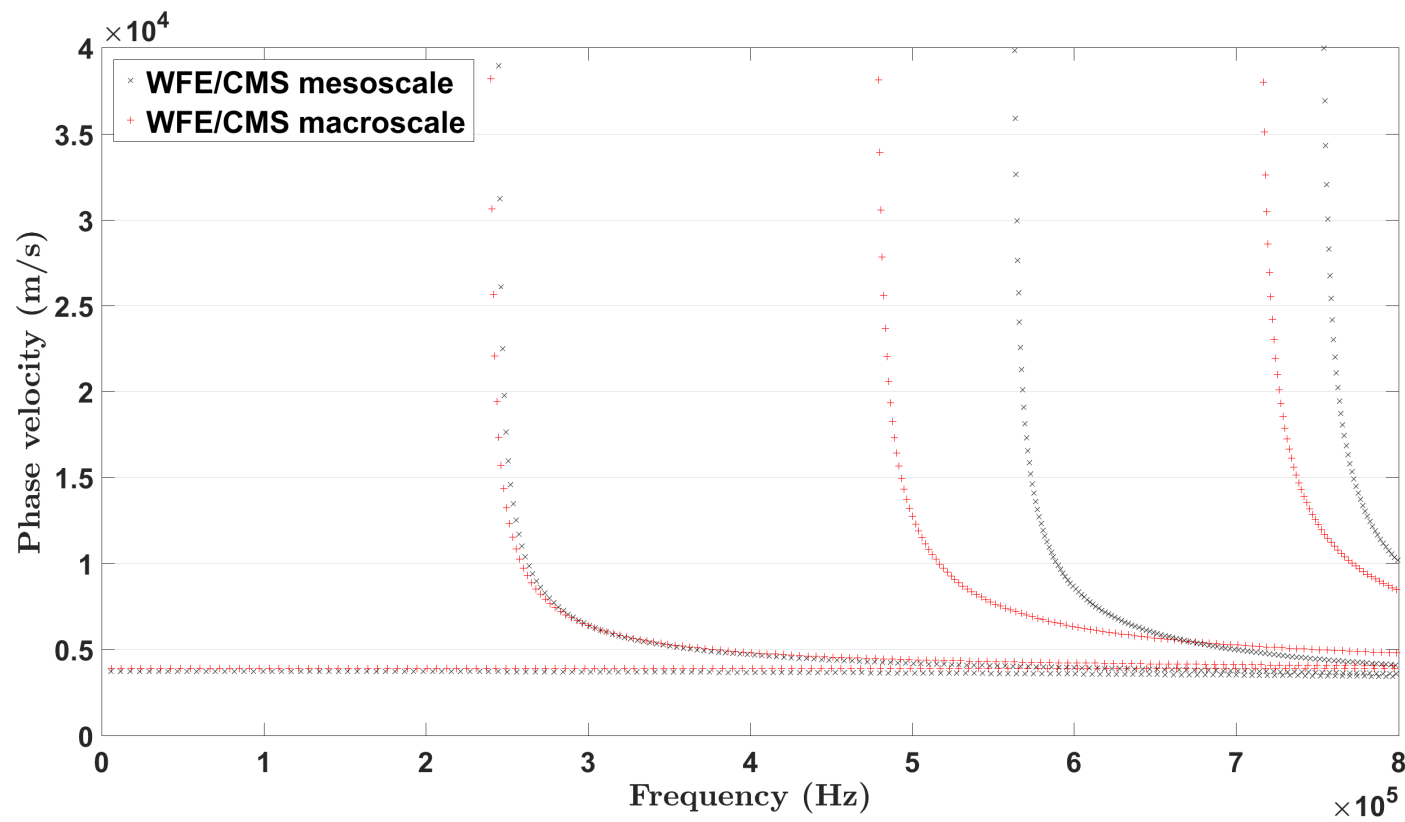

Figure 19: Phase velocity curves of the mesoscale model and macroscale model, obtained through the WFE/CMS method; 'x' mesoscale and '+' macroscale 


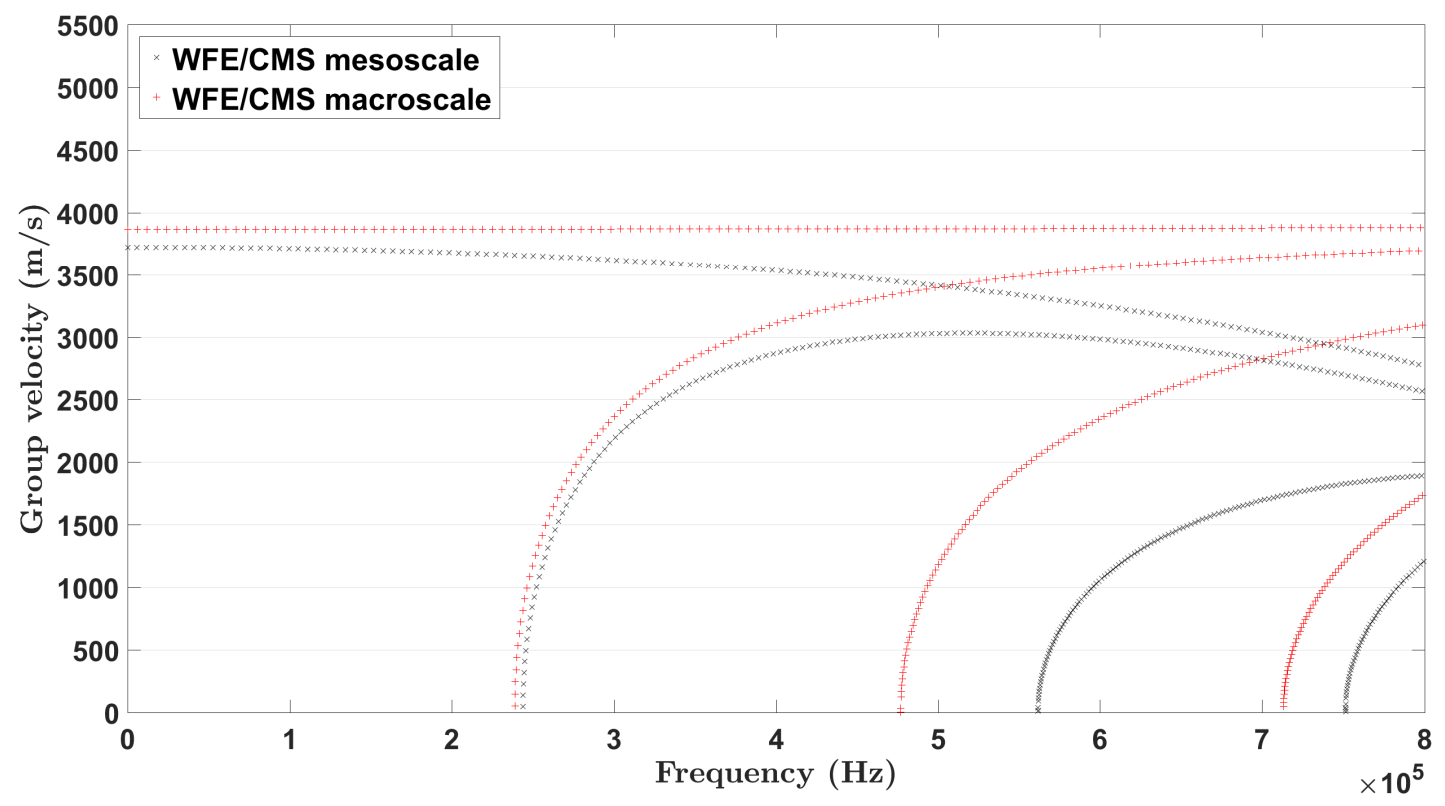

Figure 20: Group velocity curves of the mesoscale model and macroscale model, obtained through the WFE/CMS method; ' $x$ ' mesoscale and ' + ' macroscale

noticed between the cut-on frequencies of the dispersion curves of the following modes, again when comparing the same model at two different scales. The frequency of appearance for the second mode differs by $2 \%, 15 \%$ for the third mode and $5 \%$ for the forth mode.

In the context of NDE, it is of the utmost importance to know the accurate dispersion properties of the material such as the group velocity, as NDE methods often uses the time of flight of a wave to locate a damage. In the studied case (Fig.20), the relative difference between the group velocity values of the first mode of both studied scales is constantly growing and even reaches $20 \%$ at $700 \mathrm{kHz}$, which is a non-negligible difference for uses in NDE.

Figures 21 and 22 present the results obtained through the full FEM and display the results from the developed methods as well, allowing for comparison. Figure 21 presents the phase velocity results and an excellent agreement between the results from the full FE model and the developed method when the material is considered orthotropic (macroscale) can be observed, which confirms the validity of the WFE/CMS method in regard to predicting vibrational behaviour of macroscale models. When considered at a mesoscopic scale, the two models show an excellent agreement as well, the mean relative difference being of only $0.09 \%$.

When considering the group velocity (see Fig.22), a good agreement can also be observed between the results from both methods. The mean relative difference is of $0.69 \%$ for the macroscale model and of only $0.18 \%$ for the mesoscale model. These excellent agreements between the results from the widely acknowledged full FE method and the method used in this paper stand as a numerical validation of the method. 


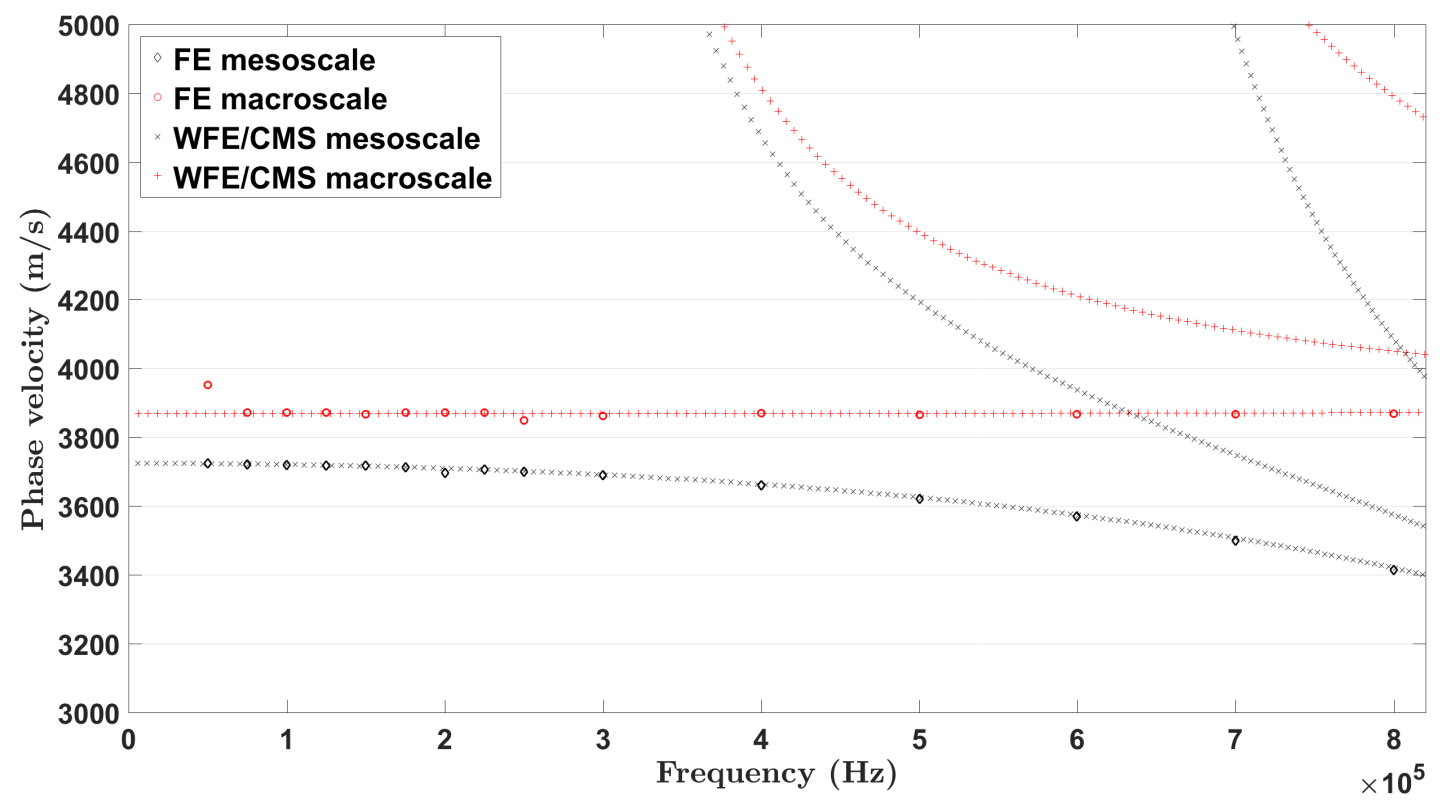

Figure 21: Zoom on the phase velocity curves of the first mode of the mesoscale model and macroscale model, comparison between results obtained through the WFE/CMS method and through the full FEM; 'x' mesoscale WFE/CMS, '+' macroscale WFE/CMS, 'diamond' mesoscale full FE and 'o' macroscale full FE

\section{Numerical investigation of a 3D weave fabric}

In this section, two models are compared. Both are models of a 3D weave as represented on the top left figure in Fig.23. One fine model of the 3D weave containing 15625 elements $(25 \times 25 \times 25)$. The second has one element in the $x$ and $y$ direction, but 25 in the $z$ direction $(1 \times 1 \times 25)$. The elements of both models need to have the same dimensions.

The dispersion curves of the first three modes from the mesoscopic model are displayed (discontinued line), and compared to the ones from the macroscopic model in Fig.24-26.

It can be observed once again very similar results when comparing the dispersion curves of the shear mode of both meso- and macroscale models (Fig.25) for frequencies smaller than the stop-band frequency.

Stop-bands are observed in the dispersion curve of the flexural mode (Fig.24) in the frequency range [2.94e5,3.17e5] $\mathrm{Hz}$, as well as in the dispersion curve of the shear mode (Fig.25) in the frequency range [4.21e5,5.61e5] Hz. Once again, the stop-bands appear when the wavelength reaches one periodicity of the unit cell.

The last three figures (see Fig.27-29) show the dispersion curves for a propagation in the $y$ direction $\left(\Theta=90^{\circ}\right)$ for the three first modes. Those are plotted for both the meso- and macroscale models. It can be seen for the flexural 


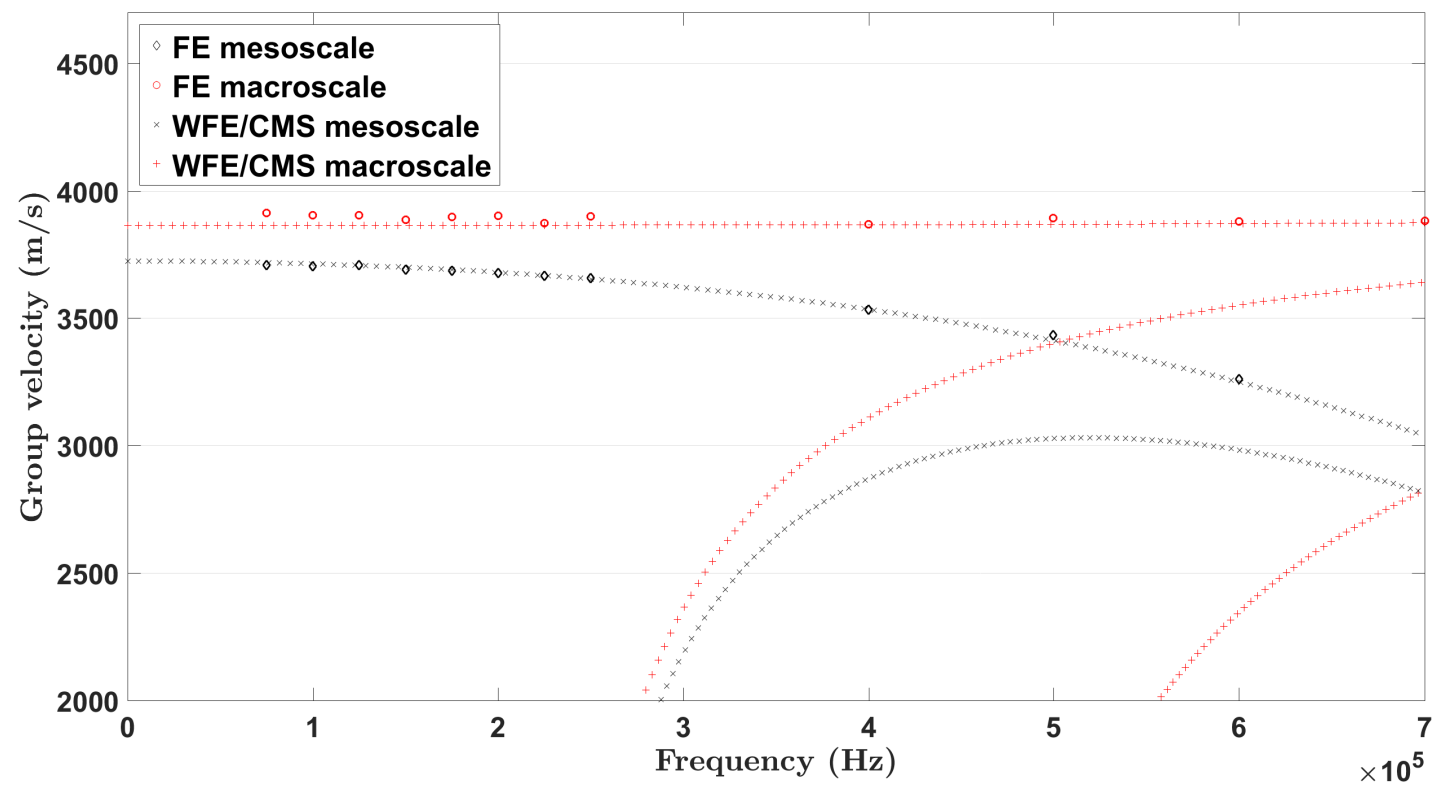

Figure 22: Zoom on the group velocity curves of the first mode of the mesoscale model and macroscale model, comparison between the WFE/CMS method and the full FEM; 'x' mesoscale WFE/CMS, ' + ' macroscale WFE/CMS, 'diamond' mesoscale full FE and 'o' macroscale full FE

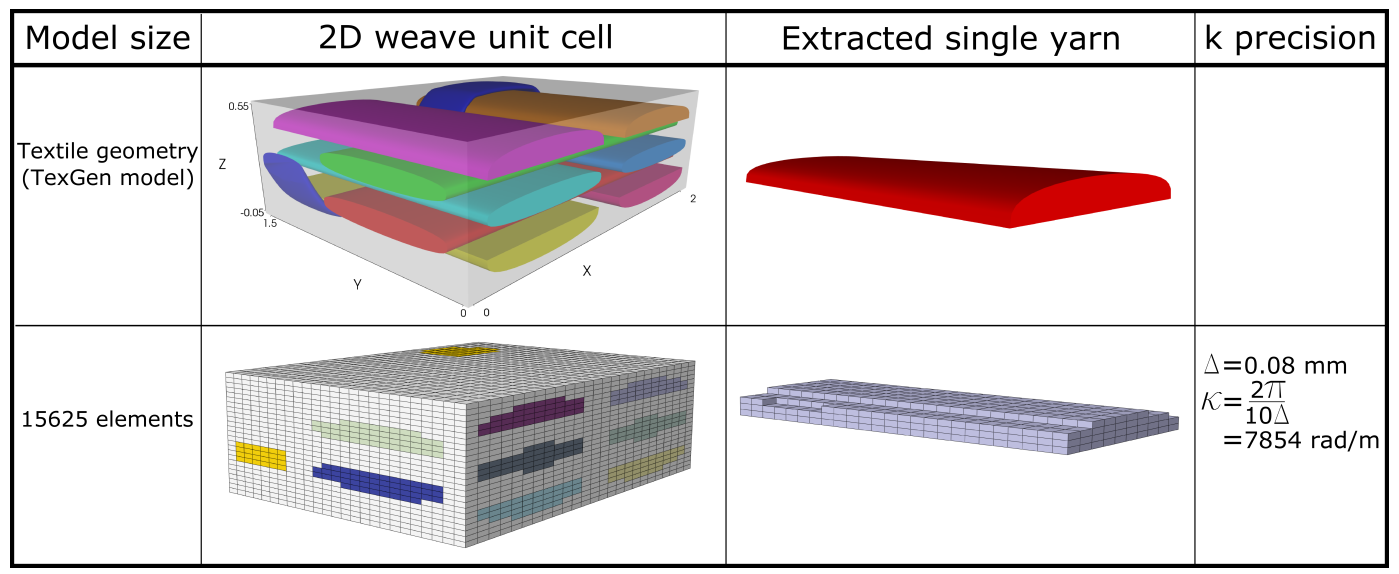

Figure 23: Meshed 3D mesoscale textile model; topological model provided by TexGen shown on the first row, mesoscale model composed of 15625 elements shown on the second and last row

and shear modes the appearance of stop-bands when the wavenumber reaches $k_{y}=\frac{1 \pi}{\Delta_{y}}, k_{y}=\frac{2 \pi}{\Delta_{y}}$ and $k_{y}=\frac{3 \pi}{\Delta_{y}}$, which means when the wavelength is equal to half the length of the unit cell or a multiple, probably because of the strong structural changes in the $y$ direction every half-length.

At last, very similar results are observed when comparing the dispersion curves of the shear mode of both meso- 


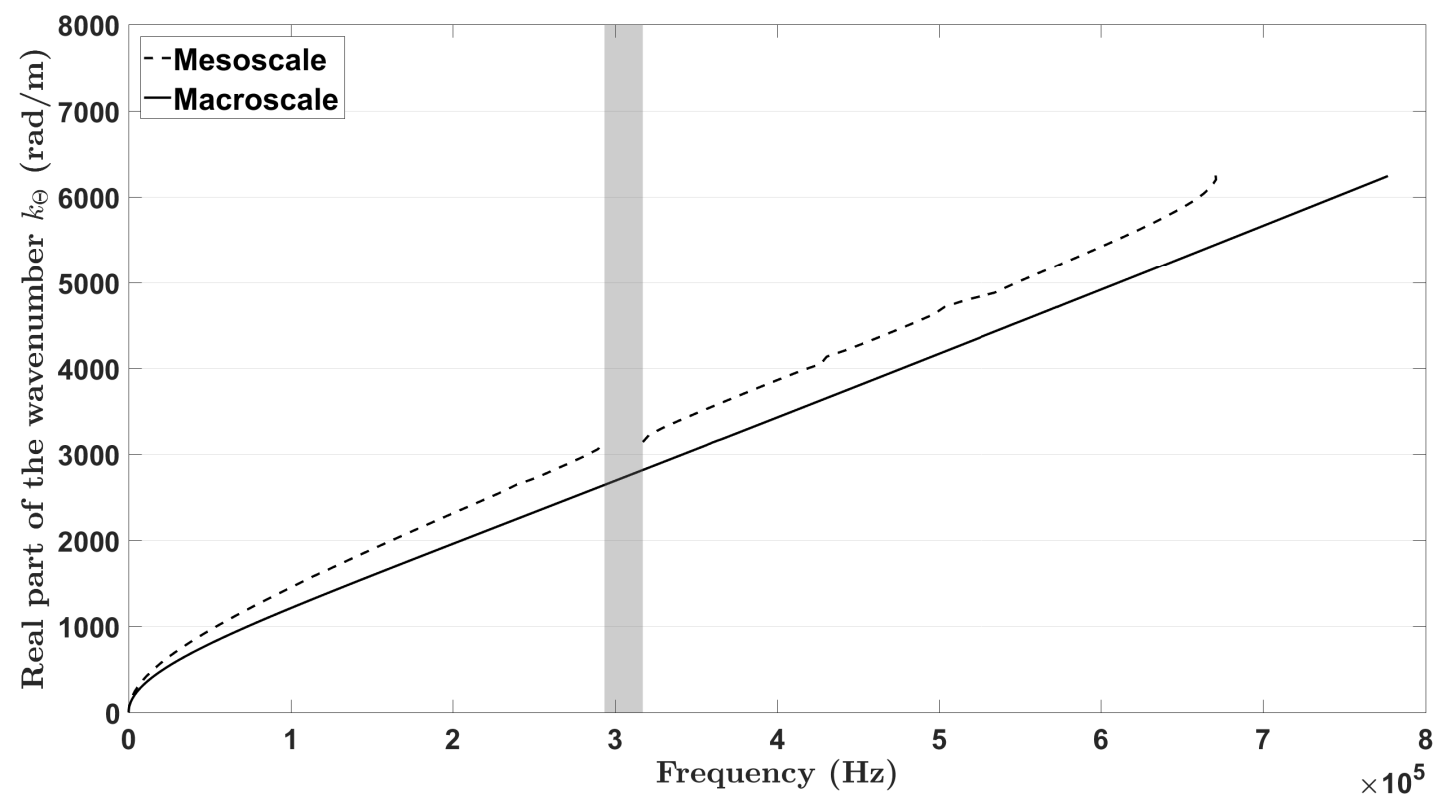

Figure 24: Dispersion curve of the flexural mode of mesoscale model and macroscale model; presenting a stop-band (grayed area) for the the mesoscale model; '- -' mesoscale model and '-' macroscale model

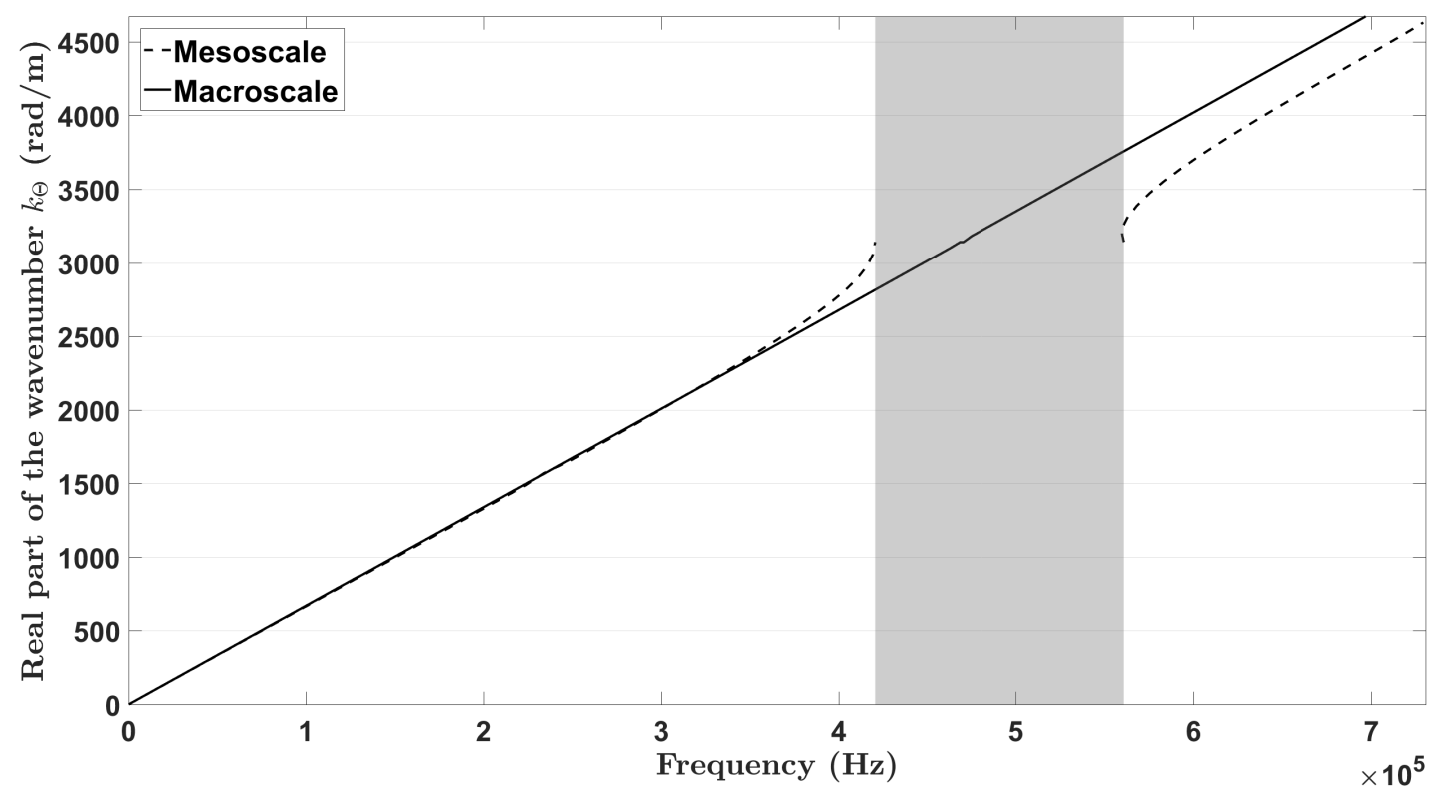

Figure 25: Dispersion curve of the shear mode of mesoscale model and macroscale model; presenting a stop-band (grayed area) for the the mesoscale model; '- -' mesoscale model and '-' macroscale model 


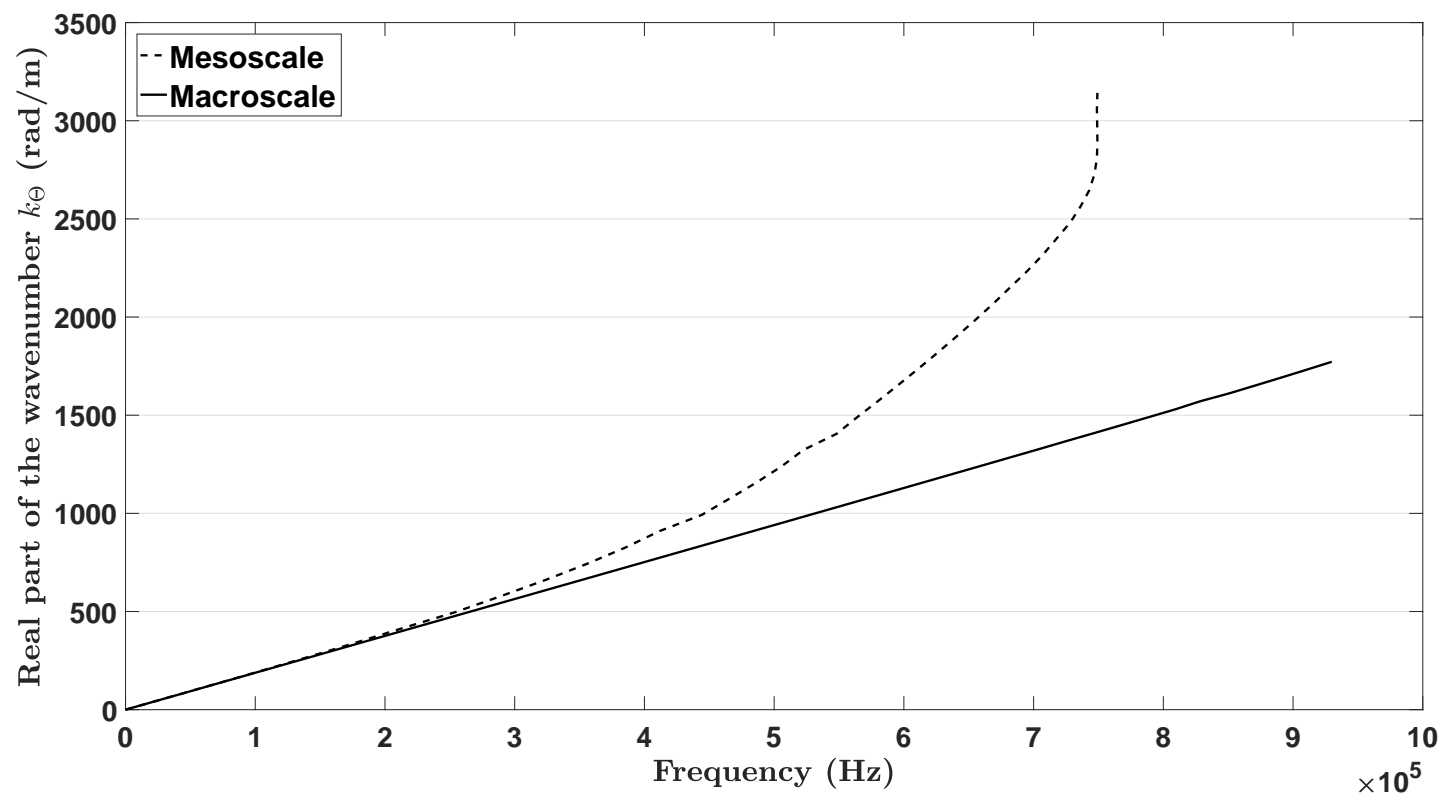

Figure 26: Dispersion curve of the extensional mode of mesoscale model and macroscale model; '- -' mesoscale model and '-' macroscale model

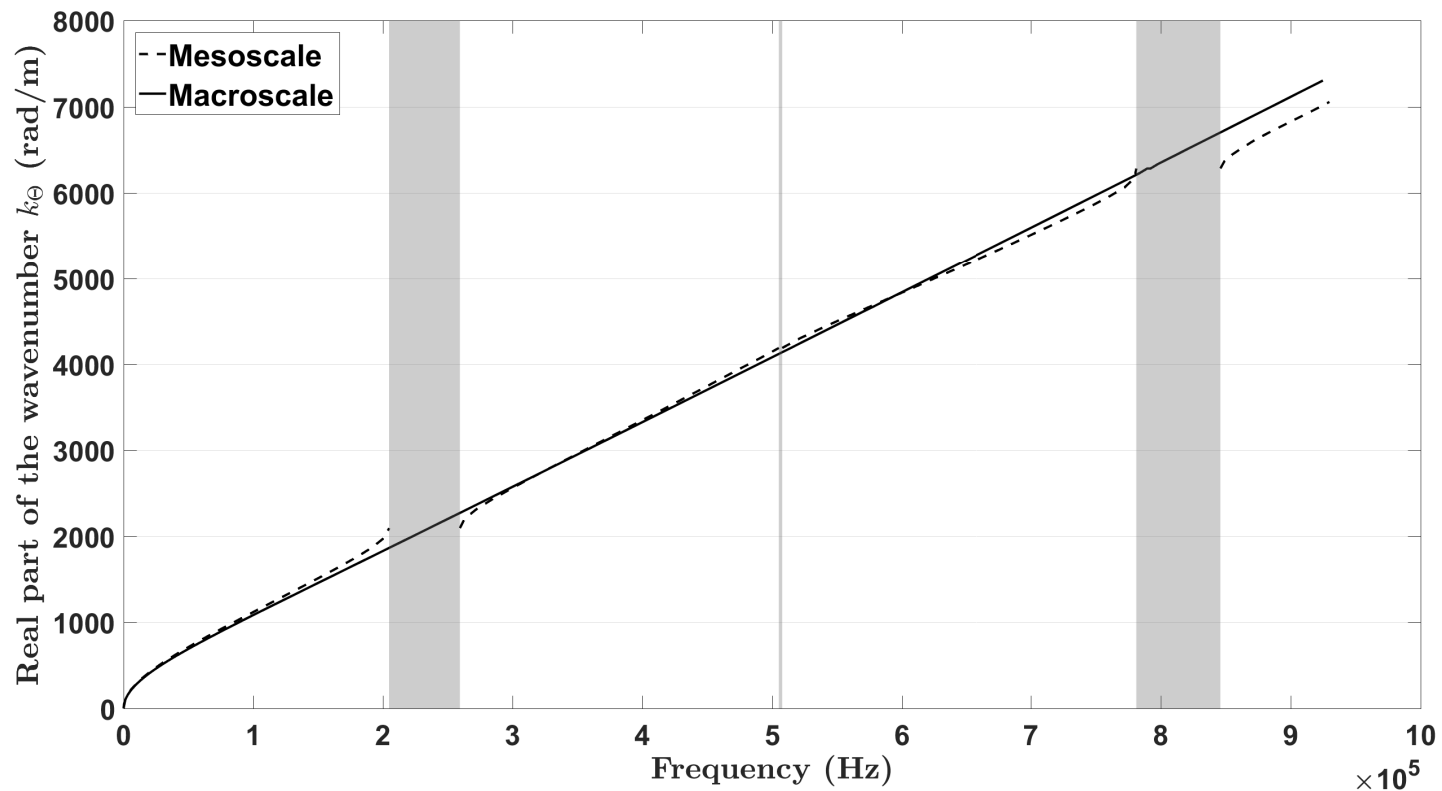

Figure 27: Dispersion curve of the flexural mode of mesoscale model and macroscale model; presenting three stopbands (grayed area) for the the mesoscale model; $\Theta=90^{\circ}$; '- -' mesoscale model and '-' macroscale model 


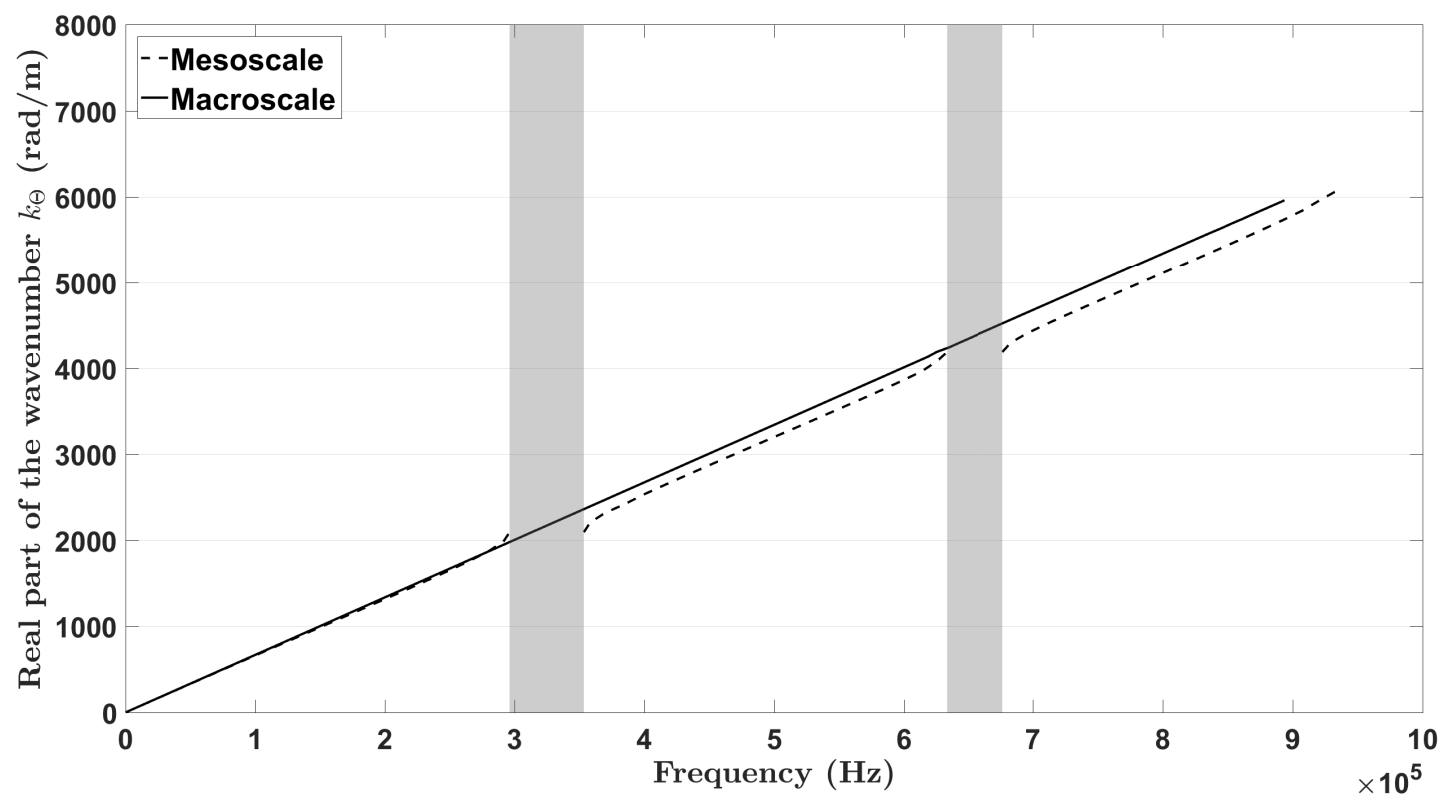

Figure 28: Dispersion curve of the shear mode of mesoscale model and macroscale model; presenting two stop-bands (grayed area) for the the mesoscale model; $\Theta=90^{\circ}$; '- -' mesoscale model and '-' macroscale model

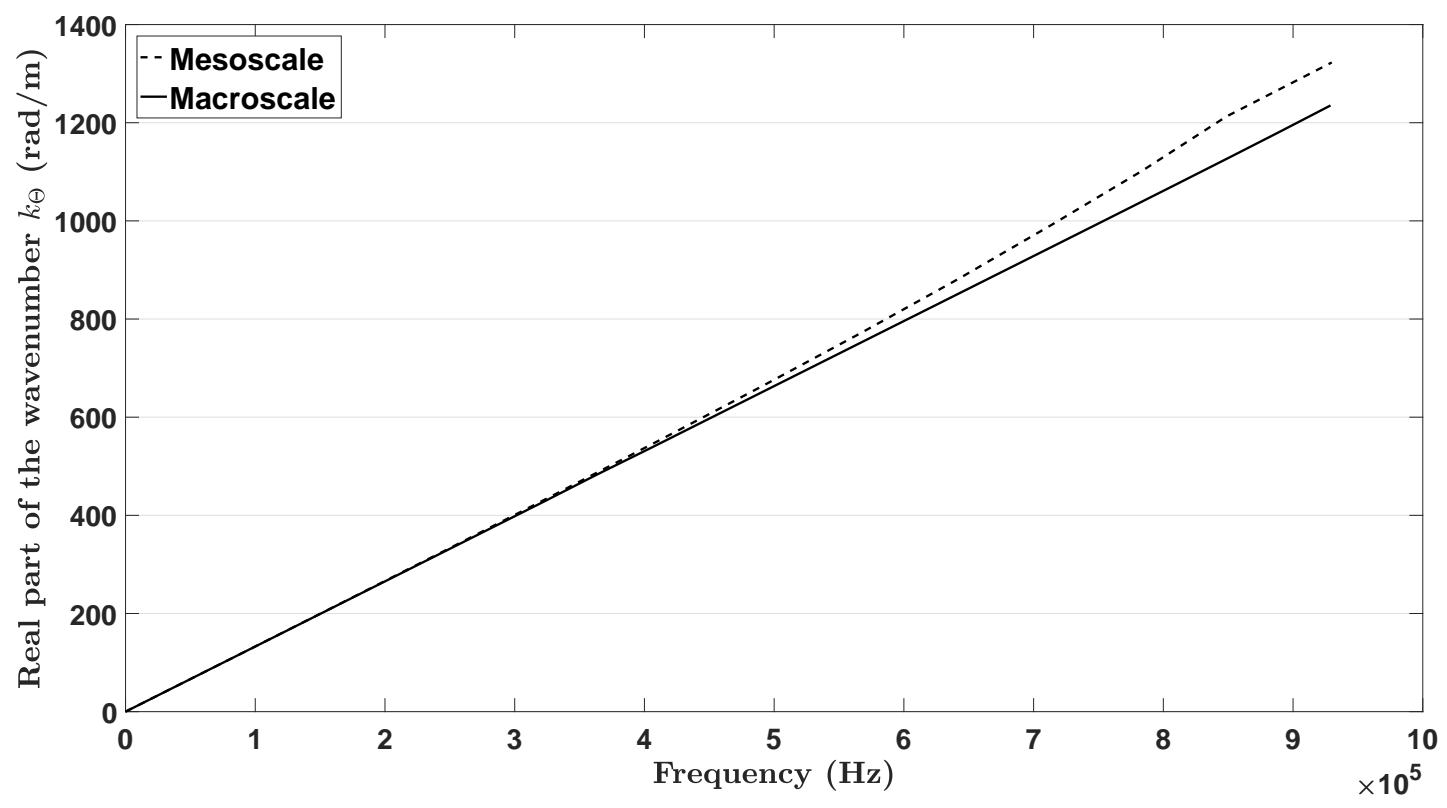

Figure 29: Dispersion curve of the extensional mode of mesoscale model and macroscale model; $\Theta=90^{\circ}$; '- -' mesoscale model and '-' macroscale model 
and macroscale models (Fig.28) for frequencies smaller than the first stop-band frequency. The mesoscale architecture of the textile seems to have a low effect on the shape of the dispersion curves of the shear mode, apart of course from the stop-bands.

\section{Conclusions}

In this paper a methodology that allows the ultrasonic wave propagation properties of arbitrary textile composites to be studied is presented. It is shown that mesoscopic scale design is needed for increased accuracy compared to the macroscopic scale calculation used in conventional studies. Two examples are presented: a $2 \mathrm{D}$ fabric weave and a 3D fabric weave. For the 2D fabric weave model, a numerical validation of the methodology is introduced. For both models, the dispersion relations are computed using the mesoscopic methodology, and compared the ones obtained with the macroscopic methodology.

- It can be seen that there are some significant differences in the dispersion relations between the two methods.

- Both methods show very similar dispersion relation for the shear mode for low frequencies.

- It appears that when studied at a mesoscopic scale, the textiles show stop-bands for certain ranges of frequency and modes.

- It appears that the strong anisotropy of the 3D weave textile in the $y$ direction creates more band-gaps.

- Despite using a few elements for the textile modeling, the results are different by only $6.5 \%$ when compared to the finest mesh model.

- Computational times are feasible while modelling the entire textile with FE would be very costly.

This paper is presented as a contribution in the field of Structural Health Monitoring in composites. The next step toward early damage detection in these structures is modelling their interactions with damages such as fibres breakage or delamination in order to build forward models. The following step will be to establish backward models.

\section{Acknowledgments}

This work is funded by the INNOVATIVE doctoral programme. The INNOVATIVE programme is partially funded by the Marie Curie Initial Training Networks (ITN) action (project number 665468) and partially by the Institute for Aerospace Technology (IAT) at the University of Nottingham.

Software engineering support was funded by the Engineering and Physical Sciences Research Council RSE Fellowship [Grant number: EP/N019040/1]. 
[1] C. Soutis, "Fibre reinforced composites in aircraft construction," Progress in Aerospace Sciences, vol. 41, no. 2, pp. 143-151, 2005.

[2] S. Mead, D. J.; Parthan, "Free wave propagation in two-dimensional periodic plates," Journal of Sound and Vibration, 1979.

[3] D. . J. Mead, "Wave propagation in continuous periodic structures: research contributions from southampton, 1964-1995," Journal of Sound and Vibration, 1996.

[4] L. Hinke, B. R. Mace, and M. J. Brennan, "Finite element analysis of waveguides," Institute of Sound and Vibration Research, 2004.

[5] C. Barbarosie and M. M. Neves, "Periodic structures for frequency filtering: analysis and optimization," Computers $\mathcal{E}$ Structures, vol. 82, no. 17-19, pp. 1399-1403, 2004.

[6] C. Droz, C. Zhou, M. N. Ichchou, and J. P. Lain, "A hybrid wave-mode formulation for the vibro-acoustic analysis of 2d periodic structures," Journal of Sound and Vibration, vol. 363, pp. 285-302, 2016.

[7] S. H. S. Gopalakrishnan, M. Ruzzene, Computational Techniques for Structural Health Monitoring. Springer, 2011.

[8] S. S. K. S. M. S. C. Soutis, "Damage detection in composite materials using lamb wave methods," Institute of Physics Publishing, 2002.

[9] V. Giurgiutiu, Structural Health Monitoring of Aerospace Composites. Academic Press, 2015.

[10] J. M. Mencik and M. N. Ichchou, "Multi-mode propagation and diffusion in structures through finite elements," European Journal of Mechanics - A/Solids, vol. 24, no. 5, pp. 877-898, 2005.

[11] B. R. Mace, D. Duhamel, M. J. Brennan, and L. Hinke, "Finite element prediction of wave motion in structural waveguides," The Journal of the Acoustical Society of America, vol. 117, no. 5, pp. 2835-2843, 2005.

[12] E. Manconi and B. R. Mace, "Modelling wave propagation in two-dimensional structures using a wave/finite element technique," Institute of Sound and Vibration Research, 2007.

[13] E. Manconi and B. R. Mace, "Wave characterization of cylindrical and curved panels using a finite element method," J Acoust Soc Am, vol. 125 , no. 1 , pp. 154-63, 2009.

[14] M. N. Ichchou, J. Berthaut, and M. Collet, "Multi-mode wave propagation in ribbed plates: Part i, wavenumber-space characteristics," International Journal of Solids and Structures, vol. 45, no. 5, pp. 1179-1195, 2008.

[15] M. N. Ichchou, J. Berthaut, and M. Collet, "Multi-mode wave propagation in ribbed plates. part ii: Predictions and comparisons," International Journal of Solids and Structures, vol. 45, no. 5, pp. 1196-1216, 2008.

[16] L. Houillon, M. N. Ichchou, and L. Jezequel, "Wave motion in thin-walled structures," Journal of Sound and Vibration, vol. 281, no. 3-5, pp. 483-507, 2005.

[17] D. Chronopoulos, M. Ichchou, B. Troclet, and O. Bareille, "Predicting the broadband response of a layered cone-cylinder-cone shell," Composite Structures, vol. 107, pp. 149-159, 2014.

[18] V. Cotoni, R. S. Langley, and P. J. Shorter, "A statistical energy analysis subsystem formulation using finite element and periodic structure theory," Journal of Sound and Vibration, vol. 318, no. 4-5, pp. 1077-1108, 2008.

[19] C. W. Zhou, J. P. Lain, M. N. Ichchou, and A. M. Zine, "Multi-scale modelling for two-dimensional periodic structures using a combined mode/wave based approach," Computers $\mathcal{E}$ Structures, vol. 154, pp. 145-162, 2015.

[20] C. Zhou, J. Lainé, M. Ichchou, and A. Zine, "Wave finite element method based on reduced model for one-dimensional periodic structures," International Journal of Applied Mechanics, vol. 7, no. 02, p. 1550018, 2015.

[21] Y. Fan, M. Collet, M. Ichchou, L. Li, O. Bareille, and Z. Dimitrijevic, "Energy flow prediction in built-up structures through a hybrid finite element/wave and finite element approach," Mechanical Systems and Signal Processing, vol. 66-67, pp. 137-158, 2016.

[22] J. M. Mencik, "A model reduction strategy for computing the forced response of elastic waveguides using the wave finite element method," Computer Methods in Applied Mechanics and Engineering, vol. 229-232, pp. 68-86, 2012.

[23] C. Droz, J. P. Lain, M. N. Ichchou, and G. Inquit, "A reduced formulation for the free-wave propagation analysis in composite structures," Composite Structures, vol. 113, pp. 134-144, 2014.

[24] G. Floquet, "Sur les equations differentielles lineaires," Ann. ENS [2], vol. 12, no. 1883, pp. 47-88, 1883.

[25] F. Bloch, "ber die quantenmechanik der elektronen in kristallgittern," Zeitschrift fr Physik, vol. 52, no. 7, pp. 555-600, 1929.

[26] L. Brillouin, Wave propagation in periodic structures: electric filters and crystal lattices. Courier Corporation, 2003. 
[27] F. R. M. De Espinosa, E. Jimenez, and M. Torres, "Ultrasonic band gap in a periodic two-dimensional composite," Physical Review Letters, vol. 80 , no. 6 , p. $1208,1998$.

[28] Y. Xiao, J. Wen, D. Yu, and X. Wen, "Flexural wave propagation in beams with periodically attached vibration absorbers: band-gap behavior and band formation mechanisms," Journal of Sound and Vibration, vol. 332, no. 4, pp. 867-893, 2013.

[29] A. M. B. Braga and G. Herrmann, "Floquet waves in anisotropic periodically layered composites," The Journal of the Acoustical Society of America, vol. 91, no. 3, pp. 1211-1227, 1992.

[30] G. Wang, D. Yu, J. Wen, Y. Liu, and X. Wen, "One-dimensional phononic crystals with locally resonant structures," Physics Letters A, vol. 327 , no. 5 , pp. 512-521, 2004.

[31] T. Kundu, S. Banerjee, and K. V. Jata, "An experimental investigation of guided wave propagation in corrugated plates showing stop bands and pass bands," The Journal of the Acoustical Society of America, vol. 120, no. 3, pp. 1217-1226, 2006.

[32] Z. C. He, X. Xiao, and E. Li, "Design for structural vibration suppression in laminate acoustic metamaterials," Composites Part B: Engineering, vol. 131, pp. 237-252, 2017.

[33] M. Cong, X. Wu, and R. Liu, "Dispersion analysis of guided waves in the finned tube using the semi-analytical finite element method," Journal of Sound and Vibration, vol. 401, pp. 114-126, 2017.

[34] A. Khelif, A. Choujaa, S. Benchabane, B. Djafari-Rouhani, and V. Laude, "Guiding and bending of acoustic waves in highly confined phononic crystal waveguides," Applied physics letters, vol. 84, no. 22, pp. 4400-4402, 2004.

[35] C. Li, D. Huang, J. Guo, and J. Nie, "Engineering of band gap and cavity mode in phononic crystal strip waveguides," Physics Letters A, vol. 377, no. 38, pp. 2633-2637, 2013.

[36] H. J. Sutherland and R. Lingle, "Geometric dispersion of acoustic waves by a fibrous composite," Journal of Composite Materials, vol. 6, no. 4, pp. 490-502, 1972.

[37] A. Apuzzo, R. Barretta, R. Luciano, F. Marotti de Sciarra, and R. Penna, "Free vibrations of bernoulli-euler nano-beams by the stress-driven nonlocal integral model," Composites Part B: Engineering, vol. 123, pp. 105-111, 2017.

[38] R. Barretta, M. Diaco, L. Feo, R. Luciano, F. M. de Sciarra, and R. Penna, "Stress-driven integral elastic theory for torsion of nano-beams," Mechanics Research Communications, vol. 87, pp. 35-41, 2018.

[39] R. Barretta, M. Čanadija, L. Feo, R. Luciano, F. Marotti de Sciarra, and R. Penna, "Exact solutions of inflected functionally graded nanobeams in integral elasticity," Composites Part B: Engineering, vol. 142, pp. 273-286, 2018.

[40] Q. Pan, Multi-scale modelling and material characterisation of textile composites for aerospace applications. PhD thesis, University of Nottingham, 2016.

[41] M. Sherburn, Geometric and mechanical modelling of textiles. PhD thesis, University of Nottingham, 2007.

[42] H. Lin, L. P. Brown, and A. C. Long, "Modelling and simulating textile structures using texgen," Advanced Materials Research, vol. 331, pp. 44-47, 2011.

[43] H. Lin, X. Zeng, M. Sherburn, A. C. Long, and M. J. Clifford, "Automated geometric modelling of textile structures," Textile Research Journal, vol. 82, no. 16, pp. 1689-1702, 2012.

[44] S. D. Green, M. Y. Matveev, A. C. Long, D. Ivanov, and S. R. Hallett, "Mechanical modelling of 3d woven composites considering realistic unit cell geometry," Composite Structures, vol. 118, pp. 284-293, 2014.

[45] L. P. Brown and M. Sherburn, "Texgen v3.9.0,” Feb. 2017. This software is currently funded by EPSRC Research Software Engineering Fellowship - Software for Textile Modelling and Simulation, EP/N019040/1.

[46] S. Li and E. Sitnikova, 1.18 An Excursion into Representative Volume Elements and Unit Cells, pp. 451-489. Oxford: Elsevier, 2018.

[47] S. Li and A. Wongsto, "Unit cells for micromechanical analyses of particle-reinforced composites," Mechanics of Materials, vol. 36, no. 7, pp. 543-572, 2004.

[48] S. Li, "Boundary conditions for unit cells from periodic microstructures and their implications," Composites Science and Technology, vol. 68, no. 9, pp. 1962-1974, 2008.

[49] S. Li, L. F. C. Jeanmeure, and Q. Pan, “A composite material characterisation tool: Unitcells," Journal of Engineering Mathematics, vol. 95, 
no. 1, pp. 279-293, 2015.

[50] M. C. C. Bampton and R. R. Craig, Jr., "Coupling of substructures for dynamic analyses,” AIAA Journal, vol. 6, pp. 1313-1319, July 1968.

[51] J. Becker and L. Gaul, "Cms methods for efficient damping prediction for structures with friction," Proceedings of the IMAC-XXVI, Orlando, 2008.

[52] R. Langley, "A note on the force boundary conditions for two-dimensional periodic structures with corner freedoms," Journal of Sound and Vibration, vol. 167, pp. 377-381, 1993.

[53] G. B. Whitham, “A note on group velocity,” Journal of Fluid Mechanics, vol. 9, no. 3, pp. 347-352, 1960.

[54] C. Droz, O. Bareille, and M. N. Ichchou, "A new procedure for the determination of structural characteristics of sandwich plates in medium frequencies," Composites Part B: Engineering, vol. 112, pp. 103-111, 2017. 


\section{Nomenclature}

\author{
$\mathbb{M}, \mathbb{C}$ and $\mathbb{K}$ \\ $t$ \\ $\omega$ \\ f \\ $\mathbf{q}$ \\ Mass, damping and stiffness matrices of the unit cell \\ Time \\ Angular frequency \\ Forcing vector for an elastic waveguide \\ Physical displacement vector for an elastic waveguide
}

$2 b_{x}, 2 b_{y}, 2 b_{z}$

$T,{ }_{B}, L, R$

$L T, R T, L B, R B$

$b d, I$

$\lambda_{x}, \lambda_{y}$

$k_{x}, k_{y}$

$P, P^{\prime}$

$\Delta x, \Delta y, \Delta z$

$u, v, w$

$U, V, W$

$\Delta u, \Delta v, \Delta w, \Delta U, \Delta V, \Delta W$

$F_{x}, F_{y}, F_{z}, F_{x y}, F_{y z}, F_{z x}$

$\boldsymbol{\Phi}_{c}$

$\boldsymbol{\Phi}_{I}$

$\boldsymbol{\Phi}_{b d}$

$p_{\Phi}$

B

$\Lambda_{L}, \Lambda_{R}$

$\rho_{m}, \rho_{f}$

$V_{m}, V_{f}, V_{t o t}$

$V_{f}^{\text {frac }}$

E

G

v

$\epsilon_{x}, \epsilon_{y}, \epsilon_{z}$

$\gamma_{x y}, \gamma_{y z}, \gamma_{z x}$

$\sigma_{x}, \sigma_{y}, \sigma_{z}$

$\tau_{x y}, \tau_{y z}, \tau_{z x}$

$\{\epsilon\}$

$\{\sigma\}$

$[S]$

Stress vector
Dimension of the modelled unit cell along $x, y$ and $z$ direction

Top, bottom, left, right sides nodes

Left-top, right-top, left-bottom, right-bottom edges nodes

Boundary and internal nodes

Propagation constant in $x$ and $y$ direction

Wavenumber in $x$ and $y$ direction

Two points in different unit cells of a textile composite

Relative position coordinates

Displacement of a point in the mesoscaled unit cell

Displacement of a point in the macroscaled unit cell

Relative displacement

Loads

Component modes

Modes associated to the internal DOFs

Static boundary modes

Reduced set of the physical internal DOFs

Craig-Bampton projection matrix

Matrices containing the propagation constants from the periodicity relation

Matrix density and fibre density of the materials used in the textile composite

Matrix volume, fibre volume and total volume of the unit cell

Fibre fraction fiber volume

Young's modulus

Shear modulus

Poisson ration

Normal strain in $x, y$ and $z$ direction

Shear strain in respectively $O x y, O y z$ and $O z x$ plan

Normal stress in $x, y$ and $z$ direction

Shear stress in respectively ${ }_{35} O x y, O y z$ and $O z x$ plan

Strain vector

Compliance matrix 